\title{
Bootstrapping ADE M-strings
}

\section{Zhihao Duan and June Nahmgoong}

School of Physics, Korea Institute for Advanced Study, Seoul 02455, Korea

E-mail: xduanz@gmail.com, junenahmgoong@gmail.com

ABstraCT: We study elliptic genera of ADE-type M-strings in 6d $(2,0)$ SCFTs from their modularity and explore the relation to topological string partition functions. We find a novel kinematical constraint that elliptic genera should follow, which determines elliptic genera at low base degrees and helps us to conjecture a vanishing bound for the refined Gopakumar-Vafa invariants of related geometries. Using this, we can bootstrap the elliptic genera to arbitrary base degree, including D/E-type theories for which explicit formulas are only partially known. We utilize our results to obtain the $6 \mathrm{~d}$ Cardy formulas and the superconformal indices for $(2,0)$ theories.

Keywords: Conformal Field Models in String Theory, Topological Strings, F-Theory

ArXiv EPrint: 2009.03626 


\section{Contents}

1 Introduction 1

$26 \mathrm{~d}(2,0)$ SCFTs and geometries 3

3 Elliptic genera of ADE M-strings $\quad 9$

3.1 Modular bootstrap from the flip symmetry 9

$\begin{array}{ll}3.2 & \text { Modular bootstrap from the vanishing bound } \\ \end{array}$

4 Applications 18

4.1 Cardy limits on $\mathbb{R}^{4} \times T^{2} \quad 18$

$4.2(2,0)$ superconformal index and $\mathcal{W}$-algebra 21

5 Concluding remarks $\quad 24$

$\begin{array}{lr}\text { A Modular forms } & 26\end{array}$

$\begin{array}{lr}\text { B Elliptic genera } & 29\end{array}$

C GV invariants

\section{Introduction}

Recently we have witnessed a revival of interest in six-dimensional quantum field theories. In particular, $6 \mathrm{~d} \mathcal{N}=(2,0)$ superconformal field theories (SCFTs) play an important role in our understanding of lower dimensional dualities [1-4]. Although still lacking Lagrangian descriptions, they are well-known to be labeled by simply-laced, i.e., ADE Lie algebras, and allow for self-dual string solitons.

Historically, 6d $(2,0)$ SCFTs were first constructed from type IIB superstring compactifications $[5,6]$. In this paper, we consider a more unifying approach, which involves F-theory [7-9]. Roughly speaking, F-theory is a non-perturbative completion of type IIB string theory, by allowing the non-constant axion-dilaton field over the moduli. Its VEV can be dictated by the complex structure of an elliptic curve, which hints at the class of Calabi-Yau (CY) manifolds having elliptic fibrations. F-theory compactification on elliptically fibered CY threefolds can engineer six-dimensional supersymmetric gauge theory. In $[10,11]$, it is conjectured that all possible six-dimensional $\mathcal{N}=(1,0)$ SCFTs can be obtained from this approach. As particular examples, $(2,0)$ SCFTs correspond to CYs with trivial elliptic fibrations.

On the other hand, CY threefolds are central objects in another area known as topological string theory. Given a CY threefold $X$, we first consider a sigma model of a twodimensional worldsheet with fixed metric mapping to $X$. Its action needs to be topologically 
twisted in order to be topological. Then we couple the system to two-dimensional gravity, i.e., integrate over all possible metrics and topologies and obtain what is known as the topological string.

Furthermore, it was discovered that for local CY threefolds, M-theory compactification engineers $5 \mathrm{~d}$ gauge theory with $\mathcal{N}=1$ supersymmetry, as an uplift of the relation between type IIA superstring and $4 \mathrm{~d}$ physics [12]. After a sequence of seminal works [13-18] proposed a refinement of topological string theory, which interprets gauge theory results in terms of geometric invariants known as refined Gopakumar-Vafa (GV) invariants. For elliptically fibered CY threefolds that engineer 5d theories having UV completions to $6 \mathrm{~d}$, the relevant gauge-theoretic quantity is precisely the elliptic genus of self-dual strings. Therefore, understanding it becomes an important task for both communities.

Currently, a plethora of ways to compute the elliptic genera for various 6d SCFTs has already been introduced. Based on their starting point, we roughly divide them into two classes and make a very partial list. From the point of view of higher dimensional gauge theories, one can utilize the brane system if it exists. For example, concerning the E-strings or M-strings, where one considers M2-branes in a chain of M5-branes with or without M9brane, the domain wall operator method [19-21] can be used to compute their elliptic genera. Also, if one works out the $2 \mathrm{~d}$ quiver gauge theories living on the self-dual strings, one can apply localization technique through the Jeffrey-Kirwan residue prescription [22, 23]. In [19, 24-30], this was successfully carried out to tackle E-strings, M-strings, some minimal SCFTs [25] and many more. From the point of view of topological strings, traditional methods such as the topological vertex or mirror symmetry are not that straightforward to apply. The non-toric topological vertex $[24,31-33]$ is developed to compute elliptic genera of Estrings, M-strings, and certain limit of elliptic genera for several other $(1,0)$ minimal SCFTs. B-model technique is explored in $[34,35]$, but restricted to the first few genus expansions. Recently, [36-39] found a powerful way to compute the elliptic genera using 6d blow-up equations, which in particular covers all the theories in the non-higgsable clusters [40].

Last but not least, since the elliptic genus is known to transform as a Jacobi modular form, one could fully explore its modular property. In other words, we write down a modular ansatz with a finite number of undetermined coefficients, discussed in detail in section 3 , then impose sufficient constraints either from gauge theory or topological string theory to fix the unknowns. This approach is often dubbed modular bootstrap [41-46], and was successfully applied to compute the elliptic genera of E-strings, M-strings, various minimial SCFTs, conformal matters, etc., at low base degrees, sometimes in the unflavored limit.

However, even though much progress has been made in understanding the $\mathcal{N}=(1,0)$ minimal SCFTs, higher rank $(2,0)$ SCFTs have not yet been fully explored in the current literature. The A-type theory has a clear brane picture as a stack of M5-branes [19]. However, elliptic genera of strings in D/E-type theories were partially studied in the massless limit only $[27,47]$. In this paper, along the line of the modular bootstrap approach, we initiate a uniform investigation of the elliptic genera of all ADE-type $(2,0)$ SCFTs with all chemical potentials fully refined. Our approach captures the elliptic genera of massive M-strings for D- and E-type, which, as far as we know, have not been written down before. ${ }^{1}$

\footnotetext{
${ }^{1}$ We notice that some elliptic genera of D4-type theories were computed in $[42,44]$.
} 
We will discuss two different ways to bootstrap the elliptic genera. The first one is based on the fact that after a circle compactification, 6d $(2,0) \mathfrak{g}$-type SCFT becomes $5 \mathrm{~d}$ maximally supersymmetric Yang-Mills (SYM) theory with gauge group $G$. Then, the 6d partition function has an expansion in terms of instanton numbers. Although the instanton partition function is unknown if $G$ is exceptional, the perturbative part is well-known. Also, from the definition of the elliptic genus, there is a kinematical constraint that we called "flip symmetry" in subsection 3.1. Combining the two, we are able to fix many elliptic genera at low base degrees.

The second approach is based on the property of refined GV invariants. They depend on the curve classes $\alpha \in H_{2}(X, \mathbb{Z})$ and two spins related to the representation of $5 \mathrm{~d}$ little group $\mathrm{SO}(4)$, so we label them by $N_{j_{-} j_{+}}^{\alpha}$. As will be explained in subsection 3.2, there exists a vanishing bound for those invariants. Namely, for a fixed $\alpha, N_{j_{-} j_{+}}^{\alpha}$ vanishes when either $j_{-}$or $j_{+}$is sufficiently large. Nevertheless, it is, in general, not easy to derive the precise bound from the geometry. In subsection 3.2 , based on the data obtained from the elliptic genera of low base degrees, we experimentally conjecture a vanishing bound and propose that it should be able to determine the elliptic genera of arbitrary base degrees in principle.

This article is organized as follows. In section 2, we give a brief review of ADE-type $(2,0)$ SCFTs, elliptic genera, topological strings and their close relationship. In section 3, we discuss two ways to compute the elliptic genera. More specifically, in subsection 3.1, we first introduce the modular ansatz then show how to combine the $5 \mathrm{~d}$ perturbative part with flip symmetry to bootstrap the elliptic genera. In subsection 3.2, we first argue the existence of vanishing condition and conjecture an empirical bound for GV invariants of related geometries. We also show how to make use of this constraint to bootstrap the elliptic genera recursively. In section 4 , we give two applications of our results: the $6 \mathrm{~d}$ Cardy formulas for all $(2,0)$ SCFTs and the $(2,0)$ superconformal index in the $\mathcal{W}$-algebra limit. In section 5 , we finish the paper with concluding remarks.

\section{$26 d(2,0)$ SCFTs and geometries}

In this section, we briefly introduce $6 \mathrm{~d} \operatorname{ADE}(2,0)$ SCFTs and the role of the self-dual strings from the point of view of F-theory. We will further discuss the supersymmetric partition function of the $6 \mathrm{~d}$ SCFTs on $\mathbb{R}^{4} \times T^{2}$ in the $\Omega$-background and the elliptic genus of the M-strings. Finally, we explain the connection of the whole story to topological strings.

To start with, we discuss the geometric construction of six-dimensional SCFTs. This is achieved through compactifying F-theory on an elliptically fibered CY threefold. In other words, we assume the CY threefold $X$ enjoys a torus fibration $E$ over a non-compact complex surface $B$,

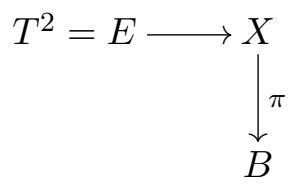

How does geometry see whether a given field theory can reach a CFT fixed point or not? Remember that although lacking Lagrangian descriptions, six-dimensional quantum field 


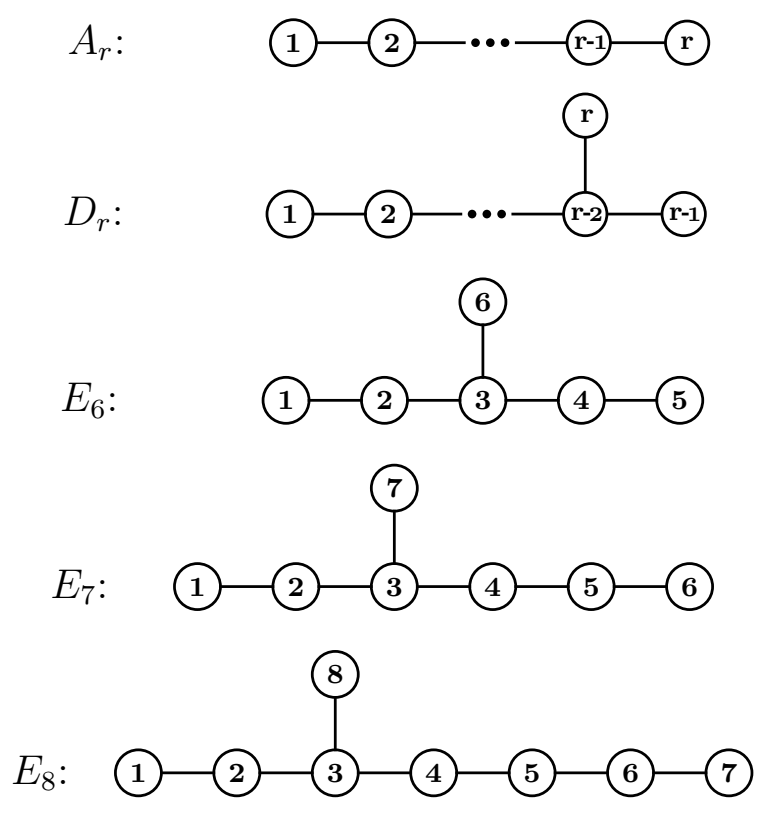

Figure 1. ADE Dynkin diagrams with numbers specifying the order.

theories have stringy solitons. This can be naturally explained in the geometric setup since a D3-brane wrapped on a two-cycle in $B$ becomes a non-critical BPS string in 6d, whose tension is proportional to the volume of the two-cycle. Such a string is called the 'self-dual string' because it inherits the self-duality condition from the D3-brane in ten dimensions. If we assume that all the compact divisors inside $B$ are contractible, then the self-dual string becomes tensionless by shrinking down all such curves, which signals a superconformal field theory.

For cases of our interest, i.e., $\mathcal{N}=(2,0)$ ADE-type SCFTs, the base $B$ in the singular or conformal limit is $\mathbb{C}^{2} / \Gamma$, with $\Gamma$ the discrete subgroups of $\mathrm{SU}(2)$. The famous McKay correspondence states that there is a one-to-one map between those subgroups and simply laced, i.e., ADE-type Lie algebras. This can be understood as follows. In the orbifold $\mathbb{C}^{2} / \Gamma$, the origin is singular, which can be resolved by a series of blow-ups. This yields exceptional curves intersecting each other according to the negative of the Cartan matrices of ADE Lie algebras. Also, notice that the resolved spaces, also known as ALE spaces, are themselves $C Y$ manifolds, so $E$ is trivially fibered over them. However, $X$ must be deformed to incorporate the M-string mass defined below, making $E$ no longer simply a trivial fibration. This means that the elliptic fiber also carries non-trivial information, which is an important reason why we choose a more general approach. We present the Dynkin diagrams of ADE Lie algebras in figure 1, where the numbers inside the node fix our convention on the ordering.

Next, bearing the above geometric picture in mind, let us put our $6 \mathrm{~d}(2,0)$ SCFTs on $\mathbb{R}^{4} \times T^{2}$ and define the BPS index. We will declare one circle of $T^{2}$ as a temporal circle, and turn on the $\Omega$-background on $\mathbb{R}^{4}$ so that the self-dual string is wrapped on $T^{2}$ and localized at the origin of $\mathbb{R}^{4}$. Then, one can define the supersymmetric partition function 
on $\mathbb{R}^{4} \times T^{2}$ as follows,

$$
Z_{\mathbb{R}^{4} \times T^{2}}\left(\tau, \epsilon_{1,2}, m, \mathbf{v}\right)=\operatorname{Tr}\left[(-1)^{F} q^{P} e^{-\mathfrak{n} \cdot \mathbf{v}} e^{-\epsilon_{1} J_{1}-\epsilon_{2} J_{2}} e^{-\left(\epsilon_{+}+m\right) R_{1}-\left(\epsilon_{+}-m\right) R_{2}}\right] .
$$

Here, $J_{1,2}$ are the two angular momenta on $\mathbb{R}^{4}$ which rotate two orthogonal planes in $\mathbb{R}^{4}$. $R_{1,2}$ are the two Cartan charges of $\mathrm{SO}(5)$ R-symmetry of $(2,0)$ supersymmetry. We introduce the corresponding chemical potentials as $\epsilon_{1,2}$ for $J_{1,2}$ and $\epsilon_{+} \pm m$ for $R_{1,2}$, with $m$ a mass deformation henceforth referred as the M-string mass and $\epsilon_{ \pm} \equiv \frac{\epsilon_{1} \pm \epsilon_{2}}{2}$. Also, $P$ is a KK momentum along the spatial circle in $T^{2}$ with the conjugate fugacity $q \equiv e^{2 \pi i \tau}$. Here, $\tau=i \frac{r_{0}}{r_{1}}$ where $r_{0}$ is the radius of the temporal circle, and $r_{1}$ is the radius of the spatial circle in $T^{2}$. Lastly, $\mathfrak{n}=\left(n_{1}, n_{2}, \ldots, n_{r}\right)$ denotes the number of the self-dual strings wrapping on each two-cycle in $B$. For this reason, we also call $\mathfrak{n}$ as a base degree. The conjugate chemical potential is given by $\mathbf{v}=\left(v_{1}, v_{2}, \ldots, v_{r}\right)$ which are proportional to the volumes of the two-cycles.

Let us take a closer look at the index. Suppose we consider a $6 \mathrm{~d}(2,0) \mathfrak{g}$-type SCFT, then the following expansion for the $\mathbb{R}^{4} \times T^{2}$ index (2.1) exists,

$$
Z_{\mathbb{R}^{4} \times T^{2}}=Z_{0} \cdot Z_{T^{2}}=Z_{0} \cdot\left(1+\sum_{\mathfrak{n} \neq 0 \in \mathbb{Z}_{\geq 0}^{r}} Z_{\mathfrak{n}} e^{-\mathfrak{n} \cdot \mathbf{v}}\right)
$$

which is valid when the string tension $\mathbf{v}$ is large. The prefactor $Z_{0}$ comes from the KK tower of BPS particles decoupled from the self-dual strings. Its form will be given in section 3. On the other hand, the expansion coefficient $Z_{\mathfrak{n}}$ denotes the contribution from the $\mathfrak{n}$ number of self-dual strings on $T^{2}$. Therefore, it is given by the elliptic genus of the $2 \mathrm{~d}(4,4)$ SCFT on the $\mathfrak{n}$ self-dual strings [19].

The elliptic genus is well known to be a weight zero Jacobi modular form (definitions can be found in appendix A). Thus what remains to determine is its index. It turns out that the index is tightly related to the anomaly polynomial of BPS strings, which we explain below.

To start with, the $6 \mathrm{~d} \mathcal{N}=(2,0)$ theory has various 't Hooft anomalies, summarized in terms of the eight-form anomaly polynomial. Then based on the anomaly inflow mechanism, $[29,48]$ successfully computed the general four-form anomaly polynomial on the $2 \mathrm{~d}$ worldsheet of BPS strings. In our case, it gets simplified,

$$
I_{4}=\sum_{a, b=1}^{r_{\mathfrak{g}}} \frac{\Omega_{a, b} n_{a} n_{b}}{2}\left(c_{2}(L)-c_{2}(R)\right)+\sum_{a=1}^{r_{\mathfrak{g}}} n_{a}\left(-\frac{1}{4} \operatorname{Tr} F_{\mathrm{SU}(2)_{m}}^{2}+c_{2}(I)\right) \text {, }
$$

with $\Omega$ the Cartan matrix of the simply-laced Lie algebras $\mathfrak{g}$ with rank $r_{\mathfrak{g}} \cdot c_{2}(L)$ and $c_{2}(R)$ the second Chern classes of two $\mathrm{SU}(2)$ bundles that split the normal bundle of strings. Moreover, we have split the $\mathrm{SO}(5)$ R-symmetry into $\mathrm{SU}(2)_{m} \times \mathrm{SU}(2)_{R}$ in (2.1), and $F_{\mathrm{SU}(2)_{m}}$ is the background field strength associated to the $\mathrm{SU}(2)_{m}$ while $c_{2}(I)$ is the second Chern class of the $\mathrm{SU}(2)_{I}$ bundle inherited from the $\mathrm{SU}(2)_{R}$. 
$I_{4}$ is responsible for a non-trivial index. Indeed, if we perform an equivariant integration in the $\Omega$-background [49] and get (we rescale the parameters to match our convention),

$$
\begin{aligned}
\mathfrak{i}_{\mathfrak{n}}= & I_{4}\left(c_{2}(L) \rightarrow-\left(\frac{\epsilon_{-}}{2 \pi i}\right)^{2}, c_{2}(R) \rightarrow-\left(\frac{\epsilon_{+}}{2 \pi i}\right)^{2},\right. \\
& \left.\frac{1}{4} \operatorname{Tr} F_{\mathrm{SU}(2)_{m}}^{2} \rightarrow-\left(\frac{m}{2 \pi i}\right)^{2}, c_{2}(I) \rightarrow-\left(\frac{\epsilon_{+}}{2 \pi i}\right)^{2}\right) \\
= & -\frac{1}{4 \pi^{2}}\left(-\sum_{a, b} \frac{\Omega_{a, b} n_{a} n_{b}}{2}\left(\epsilon_{-}^{2}-\epsilon_{+}^{2}\right)+\sum_{a} n_{a}\left(m^{2}-\epsilon_{+}^{2}\right)\right),
\end{aligned}
$$

then the elliptic genus has the following modular anomaly under an S-transformation [41],

$$
Z_{\mathfrak{n}}\left(-\frac{1}{\tau} \mid \frac{\epsilon_{1,2}}{\tau}, \frac{m}{\tau}\right)=\exp \left[2 \pi i \frac{\mathfrak{i}_{\mathfrak{n}}}{\tau}\right] \cdot Z_{\mathfrak{n}}\left(\tau \mid \epsilon_{1,2}, m\right) .
$$

Comparing (2.5) with the definition of Jacobi forms ((A.10) in the appendix A), we learn that $i_{\mathfrak{n}}$ is precisely its index.

Let us make a few comments about the existing literature. The $6 \mathrm{~d}(2,0) \mathrm{A}$-type theories are given by the worldvolume theory on $r_{\mathfrak{g}}+1$ M5-branes, and M2-branes ending on M5branes become the self-dual strings in $6 \mathrm{~d}$. With this brane construction, one can engineer the $2 \mathrm{~d}$ worldvolume theory on the $\mathfrak{n}$ self-dual strings, which is given by the A-type quiver gauge theory with gauge group $U\left(n_{1}\right) \times U\left(n_{2}\right) \times \ldots \times U\left(n_{r_{\mathfrak{g}}}\right)$ [19]. The elliptic genus of an arbitrary base degree can be computed from the localization method. However, for the D/E-type SCFTs, we do not have closed-form expressions. In [27, 47], the strings in D/E-type theories were studied with the mass parameter $m$ turned off. However, as far as we know, the fully refined elliptic genera remained unknown.

In the final part of this section, we talk about the connection to topological string theory. In order to do that, we first spell out some necessary ingredients involved.

Given a CY threefold $X$, if we denote the (complexified) Kähler parameter of two cycles in $\alpha \in H_{2}(X, \mathbb{Z})$ as $t_{\alpha}$ with its exponential $Q_{\alpha}=\exp \left(i t_{\alpha}\right)$, the genus $g$ free energy $F_{g}$ has the following expansion,

$$
F_{g}(t)=\sum_{\alpha \in H_{2}(X, \mathbb{Z})} r_{g}^{\alpha} \mathbf{Q}^{\alpha} .
$$

$r_{g}^{\alpha}$ are genus $g$ Gromov-Witten $(\mathrm{GW})$ invariants, which are in general rational numbers. Furthermore, through lifting type IIA string theory to M-theory [50-52], we can do a partial resummation over $g_{s}$ to obtain another type of expansion, ${ }^{2}$

$$
F_{\text {top }}(t)=\sum_{g \geq 0} F_{g} g_{s}^{2 g-2}=\sum_{g \geq 0} \sum_{k \geq 1} \sum_{\alpha \in H_{2}(X, \mathbb{Z})}(-1)^{g-1} I_{g}^{\alpha}\left(2 \sinh \left(k \frac{g_{s}}{2}\right)\right)^{2 g-2} \frac{\mathbf{Q}^{k \alpha}}{k} .
$$

Now $I_{g}^{\alpha}$ are always integers. They are related to M2-branes in the M-theory. From the 5d perspective, BPS M2-branes wrapped on two cycles $\alpha$ in $X$ give rise to BPS states

\footnotetext{
${ }^{2}$ We rescale the conventional definition of $g_{s}$ by $\sqrt{-1}$ to match the convention from gauge theory. Same as for $\epsilon_{ \pm}$in (2.10).
} 
in the spacetime. They are naturally labeled by two spins $j_{-}$and $j_{+}$indicating their representation under the little group $\mathrm{SO}(4)$, and $I_{g}^{\alpha}$ is related to a Witten-like index over their Hilbert space $\mathcal{H}_{\mathrm{BPS}}^{\alpha}$,

$$
\operatorname{Tr}_{\mathcal{H}_{\mathrm{BPS}}^{\alpha}}(-1)^{2 j_{+}} \mu^{2 j_{-}^{3}}=\sum_{j_{ \pm} \in \mathbb{N} / 2}(-1)^{2 j_{+}}\left(2 j_{+}+1\right) \chi_{j_{-}}(\mu) N_{j_{-} j_{+}}^{\alpha}=\sum_{g=0}^{\infty} I_{g}^{\alpha}\left(\mu^{\frac{1}{2}}+\mu^{-\frac{1}{2}}\right)^{2 g}
$$

We introduce the symbol $\chi_{j}(x)$ to denote the following Laurent polynomial of an irreducible $\mathrm{SU}(2)$ highest-weight representation with spin $j \in \mathbb{N} / 2$,

$$
\chi_{j}(x)=x^{-2 j}+x^{-2 j+2}+\cdots+x^{2 j},
$$

and $N_{j_{-} j_{+}}^{\alpha}$ is the number of multiplets for BPS states in $\mathcal{H}_{\mathrm{BPS}}^{\alpha}$ with spin $j_{-}$and $j_{+}$. In general, as we move inside the moduli space of $X, N_{j_{-} j_{+}}^{\alpha}$ may vary, but $I_{g}^{\alpha}$ remains unchanged due to the property of the index. $I_{g}^{\alpha}$ are dubbed (unrefined) Gopakumar-Vafa $(\mathrm{GV})$ invariants.

For local, i.e., non-compact CY threefolds, M-theory compactification engineers five dimensional gauge theory with $\mathcal{N}=1$ supersymmetry. Putting the five dimensional spacetime under the $\Omega$-background, we have the Nekrasov partition function $Z\left(\epsilon_{1}, \epsilon_{2}, t\right)[13,14]$, where $\epsilon_{1}$ and $\epsilon_{2}$ are two formal parameters related to the generators of the Cartan subalgebra of $s o(4)=s u(2) \times s u(2)$ through the identification $\left(\epsilon_{+}, \epsilon_{-}\right)$, with $\epsilon_{ \pm}=\frac{\epsilon_{1} \pm \epsilon_{2}}{2}$.

$Z\left(\epsilon_{1}, \epsilon_{2}, t\right)$ can be used to refine the topological string partition functions [16-18]. It is worth emphasizing that this also holds for elliptically fibered CYs, which engineer five dimensional theories having six dimensional UV completions. The upshot is that the free energy enjoys a refined GV expansion,

$$
\begin{aligned}
\mathcal{F}_{\text {ref }} & =\log \mathcal{Z}_{\text {ref }}=\log Z\left(\epsilon_{1}, \epsilon_{2}, t\right) \\
& =\sum_{2 j_{ \pm} \in \mathbb{N}} \sum_{k \geq 1} \sum_{\alpha \in H_{2}(X, \mathbb{Z})} N_{j_{-j} j_{+}}^{\alpha} \frac{(-1)^{2\left(j_{-}+j_{+}\right)} \chi_{j_{-}}\left(u^{k}\right) \chi_{j_{+}}\left(v^{k}\right)}{v^{k}+v^{-k}-u^{k}-u^{-k}} \frac{\mathbf{Q}^{k \alpha}}{k},
\end{aligned}
$$

with $u=\exp \left(-\epsilon_{-}\right), v=\exp \left(-\epsilon_{+}\right)$. Due to non-compactness, the $N_{j_{-} j_{+}}^{\alpha}$ defined earlier no longer depend on the moduli so are themselves well-defined quantities, known as refined GV invariants $[15,16,34]$. Notice that they are always non-negative since they are counting numbers of BPS states.

The connection to topological string theory lies in the duality between F-theory and M-theory [53]. It is known that F-theory compactification on $X \times S^{1}$ is dual to M-theory compactification on $X$. As discussed before, in the F-theory picture, BPS strings arise from D3-branes wrapping on curves $\Sigma_{i}$ inside $B$ and can further wrap on $T^{2}$ in the spacetime. While in the M-theory picture, they are dual to M2-branes wrapping on the same curves in $B$ and the elliptic fiber $T^{2}$ in $X$. The Kähler parameters of the rational curves are identified, while the radius of the extra $S^{1}$ in F-theory is inversely proportional to the volume of elliptic fiber in M-theory. Therefore, the fiber of $X$ not only has a complex structure $\tau$ but also acquires a (complexified) Kähler parameter $t$, which justifies the wrapping of M2-branes.

After turning on the $\Omega$-background, the partition functions of self-dual BPS strings in F-theory get identified with the refined topological string partition function $\mathcal{Z}_{\text {ref }}$ of $X$ 


\begin{tabular}{|c|c|}
\hline $\mathrm{F}\left[X \times S^{1}\right]$ & $\mathrm{M}[X]$ \\
\hline $1 / R_{S^{1}}$ & $\operatorname{Vol}\left(T^{2}\right)$ \\
\hline$q=\exp (2 \pi i \tau)$ & $q=\exp (2 \pi i t)$ \\
\hline D3-branes on $\Sigma_{i}$ and $T^{2}$ & M2-branes on $\Sigma_{i}$ and $T^{2}$ \\
\hline elliptic genus : $Z_{T^{2}}$ & partition function $: \mathcal{Z}_{\text {ref }}$ \\
\hline
\end{tabular}

Table 1. Duality between F-theory and M-theory.

in M-theory. To make the precise identification, in $Z_{\mathbb{R}^{4} \times T^{2}}$, we are only interested in the elliptic genera of BPS strings $Z_{T^{2}}(2.2)$. While corresponding in $\mathcal{Z}_{\text {ref }}$ we are only summing over two-cycles that contains a non-trivial class in $H_{2}(B, \mathbb{Z})$. We abuse our notation and still call it $\mathcal{Z}_{\text {ref }}$, hoping that no confusion will occur, ${ }^{3}$

$$
\mathcal{Z}_{\text {ref }}=1+\sum_{\mathfrak{n} \neq 0 \in \mathbb{Z}_{\geq 0}^{r}} Z_{\mathfrak{n}} e^{-\mathfrak{n} \cdot \mathbf{v}}
$$

We summarize the duality in table 1.

Last but not least, the modular anomaly can also be motivated from topological string theory. This is not essential to the main result of this paper, and we include it here just for completeness. This part can be safely skipped for an uninterested reader.

Above, what we already talked about is actually only the type-A topological string, and in fact, there exists a type-B topological string theory, based on coupling gravity to B-model on the worldsheet. In this setup, we also have a genus expansion of partition function, which now depends on the holomorphic structure rather than the Kähler structure of the CY manifold. At genus zero, the partition function is holomorphic. However, for the genus larger than zero, the anti-holomorphic part no longer decouples and gives rise to the so-called holomorphic anomaly $[54,55]$. After a careful analysis of anti-holomorphic dependence, the authors in $[54,55]$ found a set of recursive equations satisfied by the partition function, which are also known as the BCOV holomorphic anomaly equations.

In [56], it is pointed out that the holomorphic anomaly is tightly related to the modular anomaly. For cases of complex structure moduli space being one adimensional, this boils down to simply the choice of the quasimodular form $E_{2}$ versus almost holomorphic modular form $\hat{E}_{2}(\tau)$ (see appendix A) in a suitable parameterization. If we choose the quasimodular form $E_{2}$, the partition function will be holomorphic but no longer modular.

For some classes of geometries that exhibit an elliptic fibration, [57] proposed the following form of the anomaly equations,

$$
\left(\partial_{E_{2}}+\frac{1}{12} \frac{\mathfrak{n} \cdot\left(\mathfrak{n}+K_{B}\right)}{2}\right) Z_{\mathfrak{n}}=0
$$

\footnotetext{
${ }^{3}$ In fact, the two $Z_{\mathfrak{n}}$ still differ by some overall factor in general $[42,43]$, which happens to be one for $(2,0)$ theories.
} 
where the modular parameter of $E_{2}$ is identified with the complex parameter of the elliptic fiber, $\mathfrak{n}$ is now regarded as an element in $H_{2}(B, \mathbb{Z}), K_{B}$ means the canonical divisor of $B$ and the dot denotes intersection inside the base surface. In [42], this was generalized to the refined case with M-string mass turned on. In particular, for geometries related to ADE M-strings, $K_{B}$ is trivial, and they propose (we change it slightly to match our convention),

$$
\left(\partial_{E_{2}}+\frac{\pi^{2}}{3}\left[-\frac{1}{4 \pi^{2}}\left(-\frac{\mathfrak{n}^{2}}{2}\left(\epsilon_{+}^{2}-\epsilon_{-}^{2}\right)-\left(\sum_{i} n_{i}\right) \epsilon_{+}^{2}+\left(\sum_{i} n_{i}\right) m^{2}\right)\right]\right) Z_{\mathfrak{n}}=0 .
$$

By comparing (2.13) and (A.19), we learn that (2.13) motivates $Z_{\mathfrak{n}}$ to be a Jacobi form and the term inside the bracket is precisely its index. Since the curves inside the resolved ALE spaces intersect according to the negative of the corresponding Cartan matrix, we find a perfect agreement between topological string theory and gauge theory.

\section{Elliptic genera of ADE M-strings}

In this section, we obtain the elliptic genera of ADE M-strings using the modular bootstrap. In subsection 3.1, we will show that a few consistency conditions can determine the elliptic genera at low base degrees. In subsection 3.2, we study the GV invariants and their vanishing bounds, which allow us to compute the elliptic genera at an arbitrary base degree.

\subsection{Modular bootstrap from the flip symmetry}

In this subsection, we discuss some consistency conditions that the elliptic genus should follow, including the 'flip symmetry,' which is a purely kinematical constraint for the selfdual strings in $(2,0)$ theories. We will show that the elliptic genera of ADE-type M-strings can be bootstrapped with those consistency conditions up to some low base degrees. The extension to an arbitrary base degree will be discussed in subsection 3.2.

As we have discussed in section 2, the elliptic genus is a modular form with the modular parameter $\tau$, and the elliptic parameters $\epsilon_{1,2}$ and $m$. Therefore, the elliptic genus of selfdual strings can be written as follows [41],

$$
Z_{\mathfrak{n}}=\eta(\tau)^{n_{0}} \cdot \frac{\mathcal{N}_{\mathfrak{n}}\left(\tau, m, \epsilon_{1,2}\right)}{\mathcal{D}_{\mathfrak{n}}\left(\tau, \epsilon_{1,2}\right)} .
$$

Here $\eta(\tau)$ is the Dedekind eta function, and $n_{0}$ is the weight of the elliptic genus which is 0 in our case. Also, $\mathcal{N}$ is a numerator, and $\mathcal{D}$ is a denominator of the elliptic genus which will be explained in the following paragraphs.

First, let us consider the denominator structure of the elliptic genus. In the thermodynamic limit $\epsilon_{1,2} \rightarrow 0$, the free energy of the $6 \mathrm{~d} \mathbb{R}^{4} \times T^{2}$ index should have the volume divergence as $\log Z_{\mathbb{R}^{4} \times T^{2}} \propto \frac{1}{\epsilon_{1} \epsilon_{2}}$. Therefore, the elliptic genus also has a pole structure at $\epsilon_{1,2}=0$. We take our denominator to capture all those volume divergences of $\mathbb{R}^{4}$ of the elliptic genus, and it can be written in the following form [41, 42, 58],

$$
\mathcal{D}_{\mathfrak{n}}=\prod_{a=1}^{r_{\mathfrak{g}}} \prod_{k=1}^{n_{a}} \frac{\theta_{1}\left(\frac{k \epsilon_{1}}{2 \pi i}\right)}{\eta(\tau)^{3}} \frac{\theta_{1}\left(\frac{k \epsilon_{2}}{2 \pi i}\right)}{\eta(\tau)^{3}}
$$


where $r_{\mathfrak{g}}$ is the rank of the Lie algebra $\mathfrak{g}$ which is the type of the $(2,0)$ SCFT. Note that the denominator itself is a modular form, and it transforms as follows under S-duality,

$$
\mathcal{D}_{\mathfrak{n}}\left(-\frac{1}{\tau}, \frac{\epsilon_{1,2}}{\tau}\right)=\tau^{-2 \sum_{a} n_{a}} \exp \left[\frac{1}{4 \pi i \tau} \sum_{a=1}^{r_{\mathfrak{g}}} \sum_{k=1}^{n_{a}} k^{2}\left(\epsilon_{1}^{2}+\epsilon_{2}^{2}\right)\right] \cdot \mathcal{D}_{\mathfrak{n}}\left(\tau, \epsilon_{1,2}\right)
$$

which means that the denominator has the weight $w_{\mathcal{D}}=-2 \sum_{a} n_{a}$ and the index $i_{\mathcal{D}}=$ $\frac{1}{2(2 \pi i)^{2}} \sum_{a=1}^{r_{\mathfrak{g}}} \sum_{k=1}^{n_{a}} k^{2}\left(\epsilon_{1}^{2}+\epsilon_{2}^{2}\right)$.

Now, let us move on to the structure of the numerator $\mathcal{N}_{\mathfrak{n}}$. Note that it depends on the elliptic parameters $m$ and $\epsilon_{1,2}$, which are also the chemical potentials of the $\mathrm{SU}(2)$ global symmetries. Therefore, one can capture the dependence of $m$ and $\epsilon_{ \pm}$in the elliptic genus in terms of the $\mathrm{SU}(2)$ Weyl-invariant Jacobi forms: $\phi_{0,1}(\tau, z)$ and $\phi_{-2,1}(\tau, z)$. They are the modular forms which are invariant under the $\mathrm{SU}(2)$ Weyl transformation of $z$, and $\phi_{k, l}(\tau, z)$ has an index $l z^{2}$ and weight $k$. See the appendix A for detailed information. Then, one can write down the numerator in the following form,

$$
\mathcal{N}_{\mathfrak{n}}=\sum_{\mathfrak{p}} C_{\mathfrak{p}} E_{4}(\tau)^{p_{4}} E_{6}(\tau)^{p_{6}} \prod_{i=1}^{3}\left[\varphi_{-2,1}\left(\tau, \frac{z_{i}}{2 \pi i}\right)\right]^{p_{-2, i}}\left[\varphi_{0,1}\left(\tau, \frac{z_{i}}{2 \pi i}\right)\right]^{p_{0, i}} .
$$

Here, $E_{4,6}(\tau)$ are the Eisenstein series, and chemical potentials are written as $\left(z_{1}, z_{2}, z_{3}\right)=$ $\left(\epsilon_{+}, \epsilon_{-}, m\right)$. Note that we express the numerator in terms of the Eisenstein series and the $\mathrm{SU}(2)$-invariant Jacobi forms. The coefficient is denoted as $C_{\mathfrak{p}}$ which depends on the vector $\mathfrak{p}=\left(p_{4}, p_{6}, p_{-2,1}, p_{0,1}, p_{-2,2}, p_{0,2}, p_{-2,3}, p_{0,3}\right) \in\left(\mathbb{Z}_{\geq 0}\right)^{8}$. Eqs. (3.2) and (3.4) is precisely our modular ansatz for $Z_{\mathfrak{n}}$.

The elliptic genus (3.1) is determined once we can fully fix the coefficient $C_{\mathfrak{p}}$ of the numerator (3.4). Fortunately, even without knowing the 2d CFT on the self-dual string, there are some consistency conditions for the elliptic genus that we can use to fix the numerator. First, one can use the modular property (2.4) of the elliptic genus. Then, the index and the weight of the numerator is given as follows,

$$
\begin{aligned}
w_{\mathcal{N}} & =-2 \sum_{a=1}^{r_{\mathfrak{g}}} n_{a} \\
i_{\mathcal{N}} & =\frac{1}{2(2 \pi i)^{2}}\left(\epsilon_{1} \epsilon_{2} \sum_{a, b}^{r_{G}} \Omega_{a, b} n_{a} n_{b}+2\left(m^{2}-\epsilon_{+}^{2}\right) \sum_{a=1}^{r_{\mathfrak{g}}} n_{a}+\sum_{a=1}^{r_{\mathfrak{g}}} \sum_{k=1}^{n_{a}} k^{2}\left(\epsilon_{1}^{2}+\epsilon_{2}^{2}\right)\right) .
\end{aligned}
$$

From (3.5), it is straightforwards to see that the power vector $\mathfrak{p}$ should satisfy the following two relations,

$$
w_{\mathcal{N}}=-2 \sum_{i=1}^{3} p_{-2, i}+4 p_{4}+6 p_{6}, \quad i_{\mathcal{N}}=\sum_{i=1}^{3}\left(p_{-2, i}+p_{0, i}\right)\left(\frac{z_{i}}{2 \pi i}\right)^{2} .
$$

Since all $p$ 's are non-negative integers, there are only a finite number of solutions for (3.6). All the coefficient $C_{\mathfrak{p}}$ 's are zero if $\mathfrak{p}$ does not satisfy (3.6). The remaining problem is to determine the finite number of the allowed coefficients.

One way to determine those non-zero coefficients is to use the instanton expansion of the 5d maximally supersymmetric Yang-Mills theory (MSYM) with gauge group $G$, which 
is the circle compactified $6 \mathrm{~d}(2,0) \mathfrak{g}$-type theory. Until now, we considered the elliptic genus expansion (2.2) of the $6 \mathrm{~d} \mathbb{R}^{4} \times T^{2}$ index,

$$
Z_{\mathbb{R}^{4} \times T^{2}}\left(\tau, m, \epsilon_{ \pm}, \mathbf{v}\right)=Z_{0}\left(1+\sum_{\mathfrak{n}} Z_{\mathfrak{n}}\left(\tau, m, \epsilon_{ \pm}\right) e^{-\mathfrak{n} \cdot \mathbf{v}}\right)
$$

whose expansion parameter is the string fugacity $e^{-\mathbf{v}}$ and the momentum mode $Z_{0}$ is given as follows,

$$
Z_{0}=\operatorname{PE}\left[r_{\mathfrak{g}} \frac{\sinh \frac{m \pm \epsilon_{-}}{2}}{\sinh \frac{\epsilon_{1,2}}{2}} \frac{q}{1-q}\right]
$$

Here, PE stands for the plethystic exponential, defined as

$$
\mathrm{PE}\left[f\left(x_{1}, x_{2}, \cdots, x_{n}\right)\right]:=\exp \left(\sum_{k=1}^{\infty} \frac{1}{k} f\left(x_{1}^{k}, x_{2}^{k}, \cdots, x_{n}^{k}\right)\right)
$$

with $x$ 's are fugacity-like variables. Instead, one can consider a different form of the expansion as follows,

$$
Z_{\mathbb{R}^{4} \times T^{2}}\left(\tau, m, \epsilon_{ \pm}, \mathbf{v}\right)=Z_{\text {pert }}\left(1+\sum_{k=1}^{\infty} Z_{k}\left(m, \epsilon_{ \pm}, \mathbf{v}\right) q^{k}\right)
$$

where the expansion parameter is the instanton fugacity $q=e^{2 \pi i \tau}$. In the above formulation, one views the $6 \mathrm{~d} \mathfrak{g}$-type $(2,0)$ SCFTs on $\mathbb{R}^{4} \times T^{2}$ as the $5 \mathrm{~d}$ MSYM on $\mathbb{R}^{4} \times S^{1}$ with gauge group $G$. Here, the KK spectrum of the compactified circle becomes the instantons in $5 \mathrm{~d}$ [59]. $Z_{\text {pert }}$ is the perturbative partition function of the 5d MSYM given as follows [60],

$$
Z_{\text {pert }}=\mathrm{PE}\left[\frac{\sinh \frac{m \pm \epsilon_{+}}{2}}{\sinh \frac{\epsilon_{1,2}}{2}} \sum_{\alpha \in \Psi_{\mathfrak{g}}^{+}} e^{-\alpha(\mathbf{v})}\right]
$$

where $\Psi_{\mathfrak{g}}^{+}$is a set of positive roots of the Lie algebra $\mathfrak{g}$. Also, $Z_{k}$ is the $k$-instanton partition function. It can be computed from ADHM quantum mechanics when $G$ is A- or D-type [61, 62]. However, when $G$ is E-type, there are no known results for the $5 \mathrm{~d}$ MSYM instanton partition function. Since we are interested in the elliptic genus of the self-dual strings in generic $6 \mathrm{~d}(2,0)$ SCFTs including the exceptional Lie algebras, we shall not use the instanton partition function to determine the elliptic genus. Instead, we shall use the perturbative data only, by imposing the following condition,

$$
\left[Z_{0} Z_{\mathfrak{n}}\left(\tau, m, \epsilon_{ \pm}\right)\right]_{q^{0}}=\left[Z_{\text {pert }}\left(m, \epsilon_{ \pm}, \mathbf{v}\right)\right]_{e^{-\mathfrak{n} \cdot \mathbf{v}}} .
$$

Here $[f]_{q}$ denotes the coefficient of $q^{0}$ of $f$, and $[f]_{e^{-\mathfrak{n} \cdot \mathbf{v}}}$ denotes the coefficient of $e^{-\mathfrak{n} \cdot \mathbf{v}}$ of $f$.

Lastly, there is another condition, which we will call a 'flip symmetry,' that can be used to fix the elliptic genus. The flip symmetry is defined as the invariance of the elliptic genus under the following flip transformation,

$$
e^{-\epsilon_{+}} \rightarrow-e^{-\epsilon_{+}}, \quad e^{-\epsilon_{-}} \rightarrow-e^{-\epsilon_{-}}, \quad e^{-m} \rightarrow-e^{-m} .
$$




\begin{tabular}{|c|c|c|c|}
\hline Base degree & Allowed terms & Perturbative data & Flip symmetry \\
\hline$(2)$ & 44 & 38 & 6 \\
\hline$\left(\begin{array}{ll}2 & 1\end{array}\right)$ & 103 & 89 & 14 \\
\hline$\left(\begin{array}{lll}1 & 2 & 1\end{array}\right)$ & 165 & 140 & 25 \\
\hline 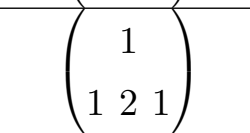 & 221 & 181 & 40 \\
\hline$\left(\begin{array}{llll} & & 1 & \\
1 & 1 & 2 & 1\end{array}\right)$ & 292 & 231 & 61 \\
\hline$\left(\begin{array}{ccccc} & & 1 & & \\
& & 1 & 2 & 1\end{array}\right)$ & 374 & 286 & 88 \\
\hline$\left(\begin{array}{llllll} & & 1 & & & \\
1 & 1 & 2 & 1 & 1 & 1\end{array}\right)$ & 468 & 346 & 122 \\
\hline$\left(\begin{array}{lllllll} & 1 & & & & \\
1 & 1 & 2 & 1 & 1 & 1 & 1\end{array}\right)$ & 575 & 411 & 164 \\
\hline
\end{tabular}

Table 2. The 'allowed terms' column denotes the number of solutions of the modular constraints (3.5). The 'perturbative data' column denotes the number of the terms whose coefficients can be fixed by the perturbative data using (3.12). The 'flip symmetry' column denotes the number of the terms whose coefficients can be further fixed by the flip symmetry using (3.13).

Under the flip transformation, the elliptic genus changes as follows,

$$
\begin{aligned}
Z_{\mathfrak{n}} & =\operatorname{Tr}\left[(-1)^{F} q^{P} e^{-\epsilon_{1} J_{1}-\epsilon_{2} J_{2}} e^{-\left(\epsilon_{+}+m\right) Q_{1}-\left(\epsilon_{+}-m\right) Q_{2}}\right] \\
& \rightarrow \operatorname{Tr}\left[e^{-2 \pi i\left(J_{1}+Q_{1}\right)} \cdot(-1)^{F} q^{P} e^{-\epsilon_{1} J_{1}-\epsilon_{2} J_{2}} e^{-\left(\epsilon_{+}+m\right) Q_{1}-\left(\epsilon_{+}-m\right) Q_{2}}\right] .
\end{aligned}
$$

Now, recall that $J_{1,2}$ are the spins of the $\mathrm{SO}(4)$ Lorentz symmetry of the tangent $\mathbb{R}^{4}$, and $Q_{1,2}$ are the spins of the unbroken $\mathrm{SO}(4)$ R-symmetry of the normal $\mathbb{R}^{4}$. Therefore, $J_{1}$ and $Q_{1}$ are integers for bosons and half-integers for fermions, i.e, $e^{-2 \pi i J_{1}}=e^{-2 \pi i Q_{1}}=(-1)^{F}$. Then, it is trivial to check that $e^{-2 \pi i\left(J_{1}+Q_{1}\right)}=1$ and the elliptic genus is invariant under the transformation (3.13)

The flip symmetry is a purely kinematic constraint in the sense that it does not depend on the microscopic details of the 2d CFT on the self-dual strings. However, it provides strong constraints when we fix the elliptic genus with the modular bootstrap. Surprisingly, the elliptic genera up to some low base degree can be completely determined from the conditions that we have mentioned so far. More precisely, the elliptic genus of the selfdual strings in $6 \mathrm{~d}(2,0)$ SCFT can be fully determined up to some base degrees from the three conditions: the modular property (3.6), the $5 d$ perturbative data (3.12), and the flip symmetry (3.13). In table 2, we tabulated the number of the coefficients of some elliptic genera whose coefficients can be totally fixed by the aforementioned three conditions. The full expression of the elliptic genera can be found in appendix B.

\footnotetext{
${ }^{4}$ This relation also plays an important role in the study of the modified indices [63-65].
} 
Although the flip symmetry can give non-trivial results for the elliptic genera, it does not give us the answer up to an arbitrarily high base degree. In the next subsection, based on the data obtained from the flip symmetry, we will establish a more powerful method that enables us to compute the elliptic genus at an arbitrary base degree.

\subsection{Modular bootstrap from the vanishing bound}

In this subsection, we first argue the existence of vanishing bound for the GV invariants. Then we present our conjecture on such a bound based on the data at low base degrees. Together with the modular ansatz introduced in the previous subsection, it can recursively bootstrap all the elliptic genera of ADE-type $(2,0)$ theories in principle. Finally, we address the issue of uniqueness for our solutions from modular bootstrap and make a comparison with the approach in subsection 3.1.

As mentioned in the introduction, a very interesting feature for the refined GV invariants $N_{j_{-} j_{+}}^{\alpha}$ is its vanishing property. But to understand that, let us first start from unrefined $\mathrm{GV}$ invariants $I_{g}^{\alpha}$. We would like to first show that for a fixed curve class $\alpha \in H_{2}(X, \mathbb{Z}), I_{g}^{\alpha}$ all vanish when $g$ is sufficiently large. If the CY manifold is a total space of canonical line bundle over a complex surface, this can be argued for using the adjunction formula [42]. However, the geometries we consider in this paper do not belong to that class, so we need a more general argument. Below, we sketch how the vanishing condition can be shown. ${ }^{5}$

In the mathematical literature, there is a class of closely related enumerative invariants called stable pair or Pandharipande-Thomas (PT) invariants $P_{n, \alpha}$ [67]. The starting point is to consider a torsion sheaf $F$ having dimension one support inside $X$, together with a non-trivial holomorphic section $s$ over it. They impose certain stability conditions, such that the zeros of sections are only a collection of points. Physically speaking, the onedimensional support corresponds to a D2-brane, and the points correspond to D0-branes. Therefore, a stable pair $(F, s)$ can be roughly thought of as a D0-D2 brane system.

The set of all possible stable pairs with a fixed holomorphic Euler characteristic $n$ and a fixed class of support $\alpha$ naturally forms a moduli space $P_{n}(X, \alpha)$. The existence of a virtual fundamental cycle was shown [67], which happens to be zero-dimensional for any CY threefold. Then the stable pair or PT invariants $P_{n, \alpha}$ just count the number of certain points inside $P_{n}(X, \alpha)$. The vanishing of $P_{n, \alpha}$ for a fixed $\alpha$ and small enough $n$ can be easily explained because a curve with a given homology class can not have arbitrarily small holomorphic Euler characteristic, rendering the moduli space empty.

On the other hand, $P_{n, \alpha}$ can be expressed as combinations of $I_{g}^{\alpha}$ [67]. For instance, for an irreducible class $\beta \in H_{2}(X, \mathbb{Z})$, they are related in terms of formal power series in $q$,

$$
\sum_{n} P_{n, \beta} q^{n}=\sum_{g \geq 0} I_{g}^{\beta} q^{1-g}(q+1)^{2 g-2} .
$$

It turns out that to satisfy the vanishing property of $P_{n, \beta}, I_{g}^{\beta}$ must also vanish when $g$ is sufficiently large. Then we argue inductively and extend the vanishing result for $I_{g}^{\beta}$ to all curve classes in $H_{2}(X, \mathbb{Z})$. In contrast, the $\mathrm{GW}$ invariants do not have such a nice property due to the presence of "bubbling phenomenon" [68].

\footnotetext{
${ }^{5}$ Another possible way of reasoning is to use the geometric model developed in [66].
} 
As for the refined GV invariants, the geometric model is more complicated so it is harder to argue from the geometry. Instead, we adopt a different strategy, through exploring the relation between the refined GV invariants and the unrefined GV invariants.

First, we need to make an important assumption: the refined GV invariants satisfy a socalled checkerboard pattern: fix a given curve class $\alpha$ and tabulate all non-zero invariants with two axes $2 j_{+}$and $2 j_{-}$. Then any two occupied blocks are either disconnected or connected through a diagonal, which makes the table resemble a checkerboard. This pattern was first noticed in [69] and holds true for all non-compact CY threefolds that the authors know of. This also applies to the geometries considered in this paper, and we refer readers to appendix $\mathrm{C}$ for examples.

Assuming the above, we next compare (2.7) and (2.10). For the reader's convenience, we repeat them below,

$$
\begin{aligned}
F_{\text {top }}(t) & =\sum_{g \geq 0} \sum_{k \geq 1} \sum_{\alpha \in H_{2}(X, \mathbb{Z})}(-1)^{g-1} I_{g}^{\alpha}\left(2 \sinh \left(k \frac{g_{s}}{2}\right)\right)^{2 g-2} \frac{\mathbf{Q}^{k \alpha}}{k}, \\
\mathcal{F}_{\text {ref }} & =\sum_{2 j_{ \pm} \in \mathbb{N}} \sum_{k \geq 1} \sum_{\alpha \in H_{2}(X, \mathbb{Z})} N_{j_{-} j_{+}}^{\alpha} \frac{(-1)^{2\left(j_{-}+j_{+}\right)} \chi_{j_{-}}\left(u^{k}\right) \chi_{j_{+}}\left(v^{k}\right)}{v^{k}+v^{-k}-u^{k}-u^{-k}} \frac{\mathbf{Q}^{k \alpha}}{k} .
\end{aligned}
$$

The unrefined limit is $\epsilon_{1}=-\epsilon_{2}=g_{s}$, from which we can deduce the following,

$$
\sum_{2 j_{ \pm} \in \mathbb{N}} N_{j_{-} j_{+}}^{\alpha}(-1)^{2\left(j_{-}+j_{+}\right)}\left(2 j_{+}+1\right) \chi_{j_{-}}\left(\mathrm{e}^{-k g_{s}}\right)=\sum_{g} I_{g}^{\alpha}(-1)^{g}\left(2 \sinh \left(k \frac{g_{s}}{2}\right)\right)^{2 g},
$$

for given $\alpha \in H_{2}(X, \mathbb{Z})$ and $k \in \mathbb{Z}_{+}$.

Thanks to the checkerboard pattern, $\left(2 j_{-}+2 j_{+}\right)$is always even or odd for a fixed curve class $\alpha$. Moreover, $\chi_{j_{-}}\left(\mathrm{e}^{-k g_{s}}\right)$ can be decomposed into a polynomial with variable $t=-\left(2 \sinh \left(k \frac{g_{s}}{2}\right)\right)^{2}$,

$$
\chi_{j_{-}}\left(\mathrm{e}^{-k g_{s}}\right)=a_{2 j_{-}} t^{2 j_{-}}+a_{2 j_{-}-1} t^{2 j_{-}-1}+\cdots+a_{0} .
$$

It is easy to show that $a_{k}>0$ for $k$ even and $a_{k}<0$ for $k$ odd always, regardless of $j_{-}$. Recall that $N_{j_{-} j_{+}}^{\alpha}$ is always a non-negative integer, this means for a given genus $g, I_{g}^{\alpha}$ can be written as a sum of non-zero $N_{j_{-} j_{+}}^{\alpha}$ with $2 j_{-} \geq g$, and each term has the same sign.

As a consequence, there must exist vanishing bound for $j_{+}$since $I_{g}^{\alpha}$ is a finite number, and also for $j_{-}$, since there is a vanishing bound for $g$ in the unrefined case. As a byproduct, this explains the pattern of alternating signs of unrefined GV invariants when we increase the genus. ${ }^{6}$

However, the bound for $j_{ \pm}$beyond which refined GV invariants all vanish depends on the details of geometry. Geometries involved here are elliptic fibrations over ALE spaces with mass deformations thereof. In practice, there are two issues. The first one is that it is not clear to us how to construct a compact embedding of $X$ into an ambient toric variety, perhaps except for the $A_{r}$ case. This prevents us from using many powerful techniques such as mirror symmetry. The other is that how to mass deform the geometry away from the

\footnotetext{
${ }^{6}$ See appendix C for some examples.
} 
trivial fibration is not known, except for the $A_{1}$ case [38]. For some relevant discussions, see $[38,39]$. Last but not least, even if one finds ways to overcome the two issues, it is perhaps equally hard to show a useful vanishing condition based on the geometry.

In short, we are far away from being able to derive an optimal vanishing bound from first principles. So instead, we guess an empirical bound for the GV invariants based on the data at low base degrees obtained from the "flip symmetry" method in subsection 3.1. The bound is not complicated, so it doesn't saturate for many cases, but we believe that it is efficient enough for practical use.

To state our conjecture, we first fix the notation. Specialize the general form (2.10) or (3.16) to our case,

$$
\mathcal{F}_{\text {ref }}=\sum_{2 j_{ \pm} \in \mathbb{N}} \sum_{k \geq 1} \sum_{\mathfrak{n}, e, \mu} \frac{N_{j_{-} j_{+}}^{\mathfrak{n}, e, \mu}}{k} \frac{(-1)^{2\left(j_{-}+j_{+}\right)} \chi_{j_{-}}\left(u^{k}\right) \chi_{j_{+}}\left(v^{k}\right)}{v^{k}+v^{-k}-u^{k}-u^{-k}} \mathbf{Q}^{k \mathfrak{n}} q^{k e} Q_{m}^{k \mu},
$$

where $\mathbf{Q}=e^{-\mathbf{v}}, q=e^{2 \pi i \tau}$ and $Q_{m}=e^{-m}$ are formal exponential of Kähler parameters associated to exceptional curves in $B$, elliptic fiber and two-cycle of M-string mass respectively. In this representation $\mathfrak{n}$ and $e$ are non-negative, but $\mu$ also takes negative values. It is possible to redefine $q$ to get rid of negative $\mu$, such that the expansion is convergent at large radius limit. However, we choose to use the present form, in order to have the symmetry $\mu \rightarrow-\mu$ in $\mathcal{F}_{\text {ref. }}$. Then due to $N_{j_{-} j_{+}}^{\mathfrak{n}, e, \mu}=N_{j_{-} j_{+}}^{\mathfrak{n}, e,-\mu}$, we only need to focus on $\mu$ being non-negative. Our conjecture for the vanishing bound is the following.

Conjecture. For elliptically fibered $C Y$ threefolds that engineer six-dimensional $\mathcal{N}=(2,0)$ $A D E$-type superconformal theories, their refined $G V$ invariants satisfy uniformly the following vanishing bound:

$$
N_{j_{-} j_{+}}^{\mathfrak{n}, e, \mu \geq 0}=0 \text { for }\left\{\begin{array}{c}
2 j_{-}>e \cdot \max \left\{n_{i}\right\}-\frac{\mu-e-1}{2} \cdot H(\mu-e-2) \\
\quad \text { or } \\
2 j_{+}>(e+2) \cdot \max \left\{n_{i}\right\}-\frac{\mu-e-1}{2} \cdot H(\mu-e-2)
\end{array}\right.
$$

On the right-hand side, $H(x)$ is the Heaviside step function,

$$
H(x)= \begin{cases}0, & \text { if } x<0 \\ 1, & \text { otherwise }\end{cases}
$$

We explain briefly how to use the above constraint to determine the partition function or elliptic genus recursively. This starts from the relation between the partition function and the free energy,

$$
\mathcal{Z}_{\text {ref }}=1+\sum_{\mathfrak{n} \neq 0 \in \mathbb{Z}_{\geq 0}^{r}} Z_{\mathfrak{n}} \mathbf{Q}^{\mathfrak{n}}=\exp \left(\mathcal{F}_{\text {ref }}\right)=\exp \left(\sum_{\mathfrak{n} \neq 0 \in \mathbb{Z}_{\geq 0}^{r}} F_{\mathfrak{n}} \mathbf{Q}^{\mathfrak{n}}\right),
$$

where in the first equality we use (2.11), and the second equality holds after we Taylor expand the exponential in terms of $\mathbf{Q}$. Therefore, for a given $\mathfrak{n}_{\alpha}=\left\{n_{1}, \cdots, n_{r}\right\}$ we have,

$$
Z_{\mathfrak{n}_{\alpha}}=F_{\mathfrak{n}_{\alpha}}+\sum_{j=2}^{\infty} \frac{1}{j !} \sum_{\substack{\mathfrak{n}_{1}, \ldots, \mathfrak{n}_{j}>\mathbf{0} \\ \sum \mathfrak{n}_{i}=\mathfrak{n}_{\alpha}}} \prod_{i=1}^{j} F_{\mathfrak{n}_{i}}
$$


Clearly, the second term in the right-hand side only involves $F_{\mathfrak{n}}$ of base degrees strictly smaller than $\mathfrak{n}_{\alpha}$. To have some feeling of this equation, we work out the expansion of the first few degrees for the E6-type,

$$
\begin{aligned}
Z_{\{1,0,0,0,0,0\}}= & F_{\{1,0,0,0,0,0\}}, \\
Z_{\{1,1,0,0,0,0\}}= & F_{\{1,1,0,0,0,0\}}+\left(F_{\{1,0,0,0,0,0\}} \cdot F_{\{0,1,0,0,0,0\}}\right) \\
Z_{\{2,1,0,0,0,0\}}= & F_{\{2,1,0,0,0,0\}}+\left(F_{\{1,1,0,0,0,0\}} \cdot F_{\{1,0,0,0,0,0\}}+F_{\{2,0,0,0,0,0\}} \cdot F_{\{0,1,0,0,0,0\}}\right) \\
& +\left(\frac{1}{2} F_{\{1,0,0,0,0,0\}}^{2} \cdot F_{\{0,1,0,0,0,0\}}\right) .
\end{aligned}
$$

For our purpose, it is better to invert the above relations,

$$
\begin{aligned}
F_{\{1,0,0,0,0,0\}}= & Z_{\{1,0,0,0,0,0\}} \\
F_{\{1,1,0,0,0,0\}}= & Z_{\{1,1,0,0,0,0\}}-\left(Z_{\{1,0,0,0,0,0\}} \cdot Z_{\{0,1,0,0,0,0\}}\right) \\
F_{\{2,1,0,0,0,0\}}= & Z_{\{2,1,0,0,0,0\}}-\left(Z_{\{1,1,0,0,0,0\}} \cdot Z_{\{1,0,0,0,0,0\}}+Z_{\{2,0,0,0,0,0\}} \cdot Z_{\{0,1,0,0,0,0\}}\right) \\
& +\left(Z_{\{1,0,0,0,0,0\}}^{2} \cdot Z_{\{0,1,0,0,0,0\}}\right) .
\end{aligned}
$$

In general we can take the logarithm of both sides of (3.22),

$$
F_{\mathfrak{n}_{\alpha}}=Z_{\mathfrak{n}_{\alpha}}+\sum_{j=2}^{\infty} \frac{1}{j !} \sum_{\substack{\mathfrak{n}_{1}, \ldots, \mathfrak{n}_{j}>\mathbf{0} \\ \sum \mathfrak{n}_{i}=\mathfrak{n}_{\alpha}}} a_{\mathfrak{n}_{1}, \ldots, \mathfrak{n}_{j}}^{\left(\mathfrak{n}_{\alpha}\right)} \prod_{i=1}^{j} Z_{\mathfrak{n}_{i}}
$$

with $a_{\mathfrak{n}_{1}, \ldots, \mathfrak{n}_{j}}^{\left(\mathfrak{n}_{\alpha}\right)}$ some integer coefficients that can be determined inductively. Clearly, the second term in the right-hand side only involves $Z_{\mathfrak{n}}$ of base degrees strictly smaller than $\mathfrak{n}_{\alpha}$.

Our strategy goes as follows. We choose $\mathfrak{n}_{\alpha}$ small enough such that all the partition functions with degree lower than $\mathfrak{n}_{\alpha}$ are known, e.g., from the "flip symmetry" method in subsection 3.1. Then we impose both the vanishing condition in the left-hand side and the modular ansatz in the right-hand side of (3.26). This turns to give enough constraints to completely fix $F_{\mathfrak{n}_{\alpha}}$ hence $Z_{\mathfrak{n}_{\alpha}}$. Increasing the degree one step at each time, we are able to determine the elliptic genus for any value of $\mathfrak{n}_{\alpha}$ in principle.

There is one important issue that needs careful discussion. Suppose we find one consistent solution to the above equation, how can we guarantee that it corresponds to the solution we want? In other words, can we show that the solution is unique? Note that if the solution is unique, then the vanishing bound must be able to fix all the coefficients in the ansatz. Happily, this point was already discussed in depth in [42]. By invoking the GV expansion and the fact that all weak Jacobi forms have a non-negative index, the authors show the following criterion [42]:

Criterion. If the index of either $\epsilon_{+}$or $\epsilon_{-}$for $Z_{\mathfrak{n}_{\alpha}}$ is smaller than -1 , then the solution to (3.26) must be unique.

Let us apply it to our situation. It so happens that the index of $\epsilon_{-}$takes the form,

$$
i_{\epsilon_{-}}=-\frac{1}{2} \mathfrak{n} \cdot \Omega \cdot \mathfrak{n}^{\mathrm{T}}
$$


where $\Omega$ is again the Cartan matrix. If we embed the simple roots into the Euclidean space, then $\Omega$ will map to the standard Euclidean inner-product and $-2 i_{\epsilon_{-}}$the norm-square of a given root. Since they are all even integral lattices, the smallest non-zero possible value for length-square is two, and the next one is four. Thus based on the criterion 3.2, only non-zero vectors with the shortest length may cause issues. For simply-laced Lie algebras, they are nothing but the positive roots. Although this could be treated generally, let us just do a case-by-case analysis here. For $\mathfrak{a}_{1}, \mathfrak{a}_{2}, \mathfrak{a}_{3}, \mathfrak{d}_{4}, \mathfrak{d}_{5}, \mathfrak{e}_{6}, \mathfrak{e}_{7}$ and $\mathfrak{e}_{8}$, there are $1,3,6$, $12,20,36,63$ and 120 in total respectively, and it is straightforward to enumerate them on a computer. Then we need to determine the index of $i_{\epsilon_{+}}$for those vectors. It turns out that in each case, for most of them, the index $i_{\epsilon_{+}}$is, fortunately, smaller than -1 , and all exceptional vectors are from low enough base degrees such that their free energies can be unambiguously determined by the "flip symmetry" method in subsection 3.1.

To summarize, combining information from both gauge theories and topological strings, we are able to show that our bootstrapping procedure gives the correct answer for the elliptic genera, provided that the conjecture 3.20 is valid.

The maximal degrees that we have computed for each theory are listed in table 3-8 for $A_{1,2,3}, D_{4,5}$, and $E_{6,7,8}$-type theories. As a consistency check, one can compare our bootstrapped elliptic genera with the known results. For A-type theories, its localization method was studied in [19], where the formula is given. We checked that the bootstrap gives exactly the same results for all the A-type elliptic genera we computed in table 3 . For D/E-type theories, its elliptic genera is currently unknown unless $m$ is turned off [27, 47]. Instead, one can use the instanton partition function of 5d D-type MSYM to test our elliptic genus. We checked that the bootstrap gives exactly the same results with the instanton partition function up to $\mathcal{O}\left(q^{1}\right)$ for all the D-type elliptic genera we computed in table 4 and 5. Especially for the $D_{4}$ case, the elliptic genera of some low base degrees are already computed in $[42,44]$. We also confirmed that our results are the same.

Lastly, for the E-type cases, there have been no known fully refined results before this paper. Therefore, we would like to emphasize that the results given in table 6,7 , and 8 are the first non-perturbative computations for the fully refined elliptic genera of the $6 \mathrm{~d}(2,0)$ E-type SCFTs.

As a final remark, let's make a comparison between the "flip symmetry" method in subsection 3.1 and the "vanishing bound" method in this subsection. On the one hand, they both rely on the modular ansatz for the partition function to start with. On the other hand, although the flip symmetry is only a kinematical constraint, it can be imposed directly on the partition function itself. Together with the perturbative part, it usually fixes most, if not all, of the coefficients in the modular ansatz at low base degrees. The rest can possibly be determined if we further demand, e.g., the GV form for the free energy, instanton partition functions expansion if available. On the other hand, the vanishing bound for GV invariants is more powerful, albeit conjectural. To make use of it, we need to subtract all the previous partition functions of smaller base degrees, which often becomes

\footnotetext{
${ }^{7}$ For example, they are not larger than the case where the degrees wrapping exceptional cycles are all equal to one.
} 
quite time-consuming in practice. This explains why we could not go very far beyond the cases already covered by the "flip symmetry" method. Also, its efficiency depends crucially on the precision of the vanishing bound.

\section{Applications}

This section discusses the two applications of the elliptic genera that we obtained from the modular bootstrap. We will mainly focus on the generalization of the results previously known only for A or D-type to all ADE theories. First, we shall compute the $6 \mathrm{~d}(2,0)$ Cardy formulas [70] on $\mathbb{R}^{4} \times T^{2}$ to all ADE-type. Second, we shall compute the $(2,0)$ superconformal index and show that it becomes the $\mathcal{W}$-algebra character in the unrefined limit for all ADE-type.

\subsection{Cardy limits on $\mathbb{R}^{4} \times T^{2}$}

In this subsection, we compute the Cardy formulas of general $6 \mathrm{~d}(2,0)$ SCFTs on $\mathbb{R}^{4} \times T^{2}$ from the elliptic genera. Ref. [70] initiated the Cardy limit study of the 6d SCFTs on $\mathbb{R}^{4} \times T^{2}$ for the $(2,0)$ A-type theory and the higher rank E-string theory. In [70], the authors computed the $6 \mathrm{~d}$ free energy by evaluating the elliptic genus summation with the continuum approximation in the Cardy limit. We will mainly follow the same method pioneered in [70], but extend their studies to $(2,0) \mathrm{D} /$ E-type SCFTs.

The Cardy limit of $\mathbb{R}^{4} \times T^{2}$ is defined as the large momenta limit, i.e., the limit where $J_{1,2}$ and $P$ are large in (2.1). In terms of the canonical ensemble, this limit can be achieved by setting the conjugate chemical potentials to be small as follows,

$$
\left|\epsilon_{1,2}\right| \ll 1, \quad|\beta| \ll 1
$$

where $\beta \equiv-2 \pi i \tau$ is a conjugate chemical potential to $P$. Now, recall that $\epsilon_{1,2}$ are the IR regulators on $\mathbb{R}^{4}$ by adjusting the effective volume as $\operatorname{vol}\left(\mathbb{R}^{4}\right) \sim \frac{1}{\epsilon_{1} \epsilon_{2}}$. Also, $\beta$ is inversely proportional to the radius of the spatial circle in $T^{2}$. Therefore, the Cardy limit (4.1) corresponds to the thermodynamic limit where the spatial volume diverges. As a result, the leading free energy in the Cardy limit should scale as $\log Z_{\mathbb{R}^{4} \times T^{2}} \sim \mathcal{O}\left(\frac{1}{\epsilon_{1} \epsilon_{2} \beta}\right)$ which is the spatial volume factor of the $6 \mathrm{~d}$ background.

In general, one may consider the complexified chemical potentials for $\epsilon_{1,2}$ and $\beta$. Such non-trivial phases of the chemical potentials are important to observe the deconfining behavior of the superconformal indices [71], including 6d SCFTs on $S^{5} \times S^{1}$ [65]. However, on $\mathbb{R}^{4} \times T^{2}$, one can observe the deconfining free energy even at the real chemical potential setting. It can be achieved by setting the $\Omega$-background parameters to be close to the self-dual point $\epsilon_{1}=-\epsilon_{2}$ by giving them different signs. Therefore, for simplicity, we shall consider the following chemical potential setting in the rest of this subsection,

$$
\epsilon_{1}>0, \quad \epsilon_{2}<0, \quad \beta>0, \quad \operatorname{Re}[m]=0 .
$$

Note that the flavor chemical potential $m$ is purely imaginary with $\operatorname{Im}[m]$ of order $\mathcal{O}(1)$. Also, we will set the tensor VEVs $\mathbf{v}$ to be sufficiently close to the origin of the tensor branch $\mathbf{v}=0$. More precisely, we will assume that $\mathbf{v} \ll \beta^{-1}$. 
We expect that the leading volume divergence $\log Z_{\mathbb{R}^{4} \times T^{2}} \sim \mathcal{O}\left(\frac{1}{\epsilon_{1} \epsilon_{2} \beta}\right)$ does not depend on the order of taking the Cardy limit (4.1). Hence, let us take the small $\beta$ limit first. In this limit, the asymptotic form of the elliptic genus can be easily computed from the modular property. Using (2.4) and (2.5), the S-dual transformation of the elliptic genus can be written as follows,

$$
Z_{\mathfrak{n}}\left(\tau \mid \epsilon_{1,2}, m\right)=\exp \left[\frac{1}{\beta}\left(\frac{\epsilon_{1} \epsilon_{2}}{2} \sum_{a, b} \Omega_{a, b} n_{a} n_{b}+\left(m^{2}-\epsilon_{+}^{2}\right) \sum_{a} n_{a}\right)\right] \cdot Z_{\mathfrak{n}}\left(-\frac{1}{\tau} \mid \frac{\epsilon_{1,2}}{\tau}, \frac{m}{\tau}\right) .
$$

Now, we consider the asymptotics of the dual elliptic genus $Z_{\mathfrak{n}}\left(-\frac{1}{\tau} \mid \frac{\epsilon_{1,2}}{\tau}, \frac{m}{\tau}\right)$ in the Cardy limit. In the right hand side of (4.3), the dual instanton fugacity $q^{D}=e^{-4 \pi^{2} / \beta}$ is much smaller than 1 . Therefore, one might guess that the dual instanton corrections in $Z_{\mathfrak{n}}\left(-\frac{1}{\tau} \mid \frac{\epsilon_{1,2}}{\tau}, \frac{m}{\tau}\right)$ can be always ignored in the Cardy limit. However, one should be careful due to the presence of other fugacities such as $e^{ \pm m / \tau}$ which can give additional growth factor that can overcome the dual instanton suppression. After carefully investigating the suppression/growth factors from each fugacities, we found that asymptotics of the dual elliptic genus $Z_{\mathfrak{n}}\left(-\frac{1}{\tau} \mid \frac{\epsilon_{1,2}}{\tau}, \frac{m}{\tau}\right)$ is given as follows,

$$
Z_{\mathfrak{n}}\left(-\frac{1}{\tau} \mid \frac{\epsilon_{1,2}}{\tau}, \frac{m}{\tau}\right)=\exp \left[\frac{-2 \pi i m(2 p+1)-4 \pi^{2}\left(p+p^{2}\right)+\mathcal{O}\left(\epsilon_{1,2}\right)}{\beta} \sum_{a} n_{a}+o\left(\beta^{-1}\right)\right]
$$

which we checked for the results given in the appendix B. Here, $p$ is an integer such that $2 \pi p<\operatorname{Im}[m]<2 \pi(p+1)$. When $p=0$, the dual elliptic genus is dominated by the dual perturbative part at $\mathcal{O}\left(\left(q^{D}\right)^{0}\right)$. When $p \neq 0$, the dominant contribution in the dual elliptic genus comes from the $\left(p+p^{2}\right) \sum n_{a}$ number of dual instantons. As we shall show later, considering the effect of dual instantons at generic $p$ is important to recover the $2 \pi i$ periodicity of $m$.

For simplicity, let us first consider the $p=0$ chamber where $0<\operatorname{Im}[m]<2 \pi$. By plugging (4.4) into (4.3), one obtains the following form,

$$
\log Z_{\mathfrak{n}}=\frac{1}{\beta}\left(\frac{\epsilon_{1} \epsilon_{2}}{2} \sum_{a, b} \Omega_{a, b} n_{a} n_{b}+m(m-2 \pi i) \sum_{a} n_{a}+\mathcal{O}\left(\epsilon_{1,2}\right)\right)+o\left(\beta^{-1}\right) .
$$

Now, let us evaluate the $6 \mathrm{~d} \mathbb{R}^{4} \times T^{2}$ index by summing over the elliptic genera as follows,

$$
\begin{aligned}
Z_{\mathbb{R}^{4} \times T^{2}} & =Z_{0} \sum_{\mathfrak{n}} Z_{\mathfrak{n}} e^{-\mathfrak{n} \cdot \mathfrak{v}} \\
& =Z_{0} \sum_{\mathfrak{n}} \exp \left[\frac{1}{\beta}\left(\frac{\epsilon_{1} \epsilon_{2}}{2} \sum_{a, b} \Omega_{a, b} n_{a} n_{b}+m(m-2 \pi i) \sum_{a} n_{a}+\mathcal{O}\left(\epsilon_{1,2}\right)\right)+o\left(\beta^{-1}\right)\right] .
\end{aligned}
$$

Note that the effect of the string fugacity $e^{-\mathfrak{n} \cdot \mathbf{v}}$ is subleading since we assumed that $\mathbf{v} \ll$ $\beta^{-1}$. Also, the summation is convergent since $\operatorname{det}\left(\frac{\epsilon_{1} \epsilon_{2}}{\beta} \Omega\right)<0$. Now, let us further take the remaining Cardy limit, i.e., $\epsilon_{1,2} \rightarrow 0$ limit. We take a continuum approximation of the string number by defining following continuous variables,

$$
x_{a} \equiv-\epsilon_{1} \epsilon_{2} n_{a} .
$$


Although $n_{a}$ is a discrete variable with $\Delta n_{a}=1, x_{a}$ can be viewed as an almost continuous variable since $\Delta x_{a}=-\epsilon_{1} \epsilon_{2} \ll 1$ in the Cardy limit. Then, the elliptic genus summation (4.6) can be approximated to the integral as follows,

$Z_{\mathbb{R}^{4} \times T^{2}}=Z_{0} \int_{0}^{\infty}\left[\prod_{a=1}^{r_{\mathfrak{g}}} d x_{a}\right] \exp \left[\frac{1}{\epsilon_{1} \epsilon_{2} \beta}\left(\frac{1}{2} \sum_{a, b} \Omega_{a, b} x_{a} x_{b}+m(2 \pi i-m) \sum_{a} x_{a}\right)+o\left(\frac{1}{\epsilon_{1} \epsilon_{2} \beta}\right)\right]$.

The free energy $\log Z_{\mathbb{R}^{4} \times T^{2}}$ can be evaluated from the saddle point approximation of the above integral. The peak of the Gaussian is located at

$$
x_{a}=m(m-2 \pi i) \sum_{b}\left(\Omega^{-1}\right)_{a, b}+\mathcal{O}\left(\epsilon_{1,2}\right) .
$$

Here, notice that the vector $\sum_{b}\left(\Omega^{-1}\right)_{a, b} \mathbf{e}_{b}=\rho$ is the Weyl vector of the Lie algebra $\mathfrak{g}$, i.e. $\rho=\frac{1}{2} \sum_{\alpha \in \Psi_{\mathfrak{g}}^{+}} \alpha$. For the simply-laced Lie algebras, the Weyl vector is given as follows,

$$
\begin{aligned}
2 \rho & =\sum_{a=1}^{r} a(r+1-a) \mathbf{e}_{a}, & & \mathfrak{g}=A_{r} \\
& =\sum_{a=1}^{r-2} a(2 r-1-a) \mathbf{e}_{a}+\frac{r(r-1)}{2}\left(\mathbf{e}_{r-1}+\mathbf{e}_{r}\right), & & \mathfrak{g}=D_{r} \\
& =(16,30,42,30,16,22), & & \mathfrak{g}=E_{6} \\
& =(34,66,96,75,52,27,49), & & \mathfrak{g}=E_{7} \\
& =(92,182,270,220,168,114,58,136), & & \mathfrak{g}=E_{8} .
\end{aligned}
$$

In the Cardy limit, the string number $\mathfrak{n}$ has a non-zero VEV on the tensor branch, given by $\left\langle n_{a}\right\rangle=-\frac{x_{a}}{\epsilon_{1} \epsilon_{2}}[70]$. Therefore, the Weyl vector of $\mathfrak{g}$ determines the distribution of the self-dual strings in $6 \mathrm{~d}(2,0) \mathfrak{g}$-type SCFT in the Cardy limit.

The contribution of the elliptic genera is localized near the peak (4.9), and the leading free energy can be obtained from the value of the integrand at the peak. As a result, we obtain the following free energy,

$$
\log Z_{\mathbb{R}^{4} \times T^{2}} \simeq \log Z_{0}-\frac{\sum_{a, b}\left(\Omega^{-1}\right)_{a, b}}{2} \frac{m^{2}(2 \pi i-m)^{2}}{\epsilon_{1} \epsilon_{2} \beta}
$$

where we ignored the subleading corrections in the Cardy limit. From the expression of $Z_{0}$ given in (3.8), one can compute that $\log Z_{0}=-\frac{r_{\mathfrak{g}}}{24} \frac{m^{2}(2 \pi i-m)^{2}}{\epsilon_{1} \epsilon_{2} \beta}$ [72]. Also, the group theoretic constant $\sum_{a, b}(\Omega)_{a, b}^{-1}$ is equal to $\frac{h_{\mathfrak{g}}^{\vee} d_{\mathfrak{g}}}{12}$ where $h_{\mathfrak{g}}^{\vee}$ is a dual Coxeter number and $d_{\mathfrak{g}}^{\vee}$ is a dimension of the Lie algebra $\mathfrak{g}$. Therefore, we obtain the Cardy formula of $6 \mathrm{~d}(2,0)$ $\mathfrak{g}$-type theory on $\mathbb{R}^{4} \times T^{2}$ as follows,

$$
\log Z_{\mathbb{R}^{4} \times T^{2}} \simeq-\frac{h_{\mathfrak{g}}^{\vee} d_{\mathfrak{g}}+r_{\mathfrak{g}}}{24} \frac{m^{2}(2 \pi i-m)^{2}}{\epsilon_{1} \epsilon_{2} \beta} .
$$

For the simply-laced Lie algebra, the overall constant $h_{\mathfrak{g}}^{\vee} d_{\mathfrak{g}}+r_{\mathfrak{g}}$ is given as follows,

$$
\begin{aligned}
h_{\mathfrak{g}}^{\vee} d_{\mathfrak{g}}+r_{\mathfrak{g}} & =(r+1)^{3}-1, & & \mathfrak{g}=A_{r} \\
& =4 r^{3}-6 r^{2}+3 r, & \mathfrak{g} & =D_{r} \\
& =942,2401,7448, & & \mathfrak{g}=E_{6,7,8}
\end{aligned}
$$


Note that in our chemical potential setting (4.2) and $0<\operatorname{Im}[m]<2 \pi$, we can see that $\log Z \gg 1$, which signals the macroscopic number of deconfining degrees of freedom. The above result is a straightforward generalization of the $(2,0)$ A-type formula in [70] to general ADE-type theories. The overall factor of the free energy $h_{\mathfrak{g}}^{\vee} d_{\mathfrak{g}}+r_{\mathfrak{g}}$ was also observed in the 6d Cardy formula on $S^{5} \times S^{1}$ [65], which explains the black hole entropy in the dual gravity.

Lastly, let us consider the free energy in the general chamber of $m$ given by $2 \pi p<$ $\operatorname{Im}[m]<2 \pi(p+1)$. In this chamber, one should use a general form (4.4) with $p \neq 0$. Inserting (4.4) to (4.3) yields,

$$
\log Z_{\mathfrak{n}}=\frac{1}{\beta}\left(\frac{\epsilon_{1} \epsilon_{2}}{2} \sum_{a, b} \Omega_{a, b} n_{a} n_{b}+(m-2 \pi i p)(m-2 \pi i(p+1)) \sum_{a} n_{a}+\mathcal{O}\left(\epsilon_{1,2}\right)\right)+o\left(\beta^{-1}\right) .
$$

After following the similar calculations explained so far, we obtain the following $6 \mathrm{~d}$ free energy,

$$
\log Z_{\mathbb{R}^{4} \times T^{2}} \simeq-\frac{h_{G}^{\vee} d_{G}+r_{G}}{24} \frac{(m-2 \pi i p)^{2}(2 \pi i(p+1)-m)^{2}}{\epsilon_{1} \epsilon_{2} \beta}
$$

One can see that the above expression is periodic under $m \sim m+2 \pi i$. Therefore, as we emphasized, the instanton correction in the dual elliptic genus (4.4) is essential to recover the periodicity of $m$.

\section{$4.2(2,0)$ superconformal index and $\mathcal{W}$-algebra}

In this subsection, we compute the superconformal indices of the $6 \mathrm{~d}(2,0)$ SCFTs from the elliptic genus method. We will focus on the unrefined limit of the chemical potentials where the superconformal indices are expected to be reduced to the $\mathcal{W}$-algebra character [73]. We show that our results agree with the prediction, including E-type cases.

Let us briefly review the superconformal index of $6 \mathrm{~d}(2,0)$ SCFT. See [74] for a detailed review. The bosonic part of the $6 \mathrm{~d}(2,0)$ superconformal algebra is given by $\mathrm{SO}(6,2)$ conformal symmetry and $\mathrm{SO}(5)$ R-symmetry. Let us denote $E$ and $J_{1,2,3}$ as the charges for $\mathrm{SO}(6,2)$ and $Q_{1,2}$ as the charges for $\mathrm{SO}(5)$, which are all normalized to be $\pm \frac{1}{2}$ for spinors. We take a defining supercharge of the index to give the BPS bound $E \geq 2 Q_{1}+2 Q_{2}+J_{1}+$ $J_{2}+J_{3}$. Then, the superconformal index is defined as follows,

$$
I=\operatorname{Tr}\left[(-1)^{F} e^{-\omega_{1} J_{1}-\omega_{2} J_{2}-\omega_{3} J_{3}} e^{-\Delta_{1} Q_{1}-\Delta_{2} Q_{2}}\right]
$$

where the trace is taken over the Hilbert space of the radially quantized 6d SCFT on $S^{5} \times \mathbb{R}_{\text {time. }}$. The chemical potentials are constrained by $\Delta_{1}+\Delta_{2}-\omega_{1}-\omega_{2}-\omega_{3}=0$ to preserve the supersymmetry. We will use the notation $\Delta_{R}=\Delta_{1}+\Delta_{2}$ and $\Delta_{L}=\Delta_{1}-\Delta_{2}$ in this subsection.

The $6 \mathrm{~d}$ superconformal index was studied from localization of 5d MSYM on $S^{5}$ [75-81]. The path integral on $S^{5}$ is localized at the three fixed points, and the contribution from each fixed point is given by the $\mathbb{R}^{4} \times T^{2}$ index on the tensor branch. The full $S^{5} \times S^{1}$ index can be obtained from the three copies of $\mathbb{R}^{4} \times T^{2}$ indices as follows [74],

$$
I=\frac{e^{-E_{0}-S_{\mathrm{bkgd}}}}{W_{\mathfrak{g}} \sqrt{\left|\Omega_{\mathfrak{g}}\right|}} \int\left[\prod_{a=1}^{r} d v_{a}\right] e^{-S_{0}} \hat{Z}_{[1]} \hat{Z}_{[2]} \hat{Z}_{[3]} .
$$


Let us explain the various factors in (4.17). First, $\hat{Z}_{[k]}$ is the Weyl-symmetrized $\mathbb{R}^{4} \times T^{2}$ index. It is basically same with the $\mathbb{R}^{4} \times T^{2}$ index in (3.10) discussed in this paper so far, but multiplied with the Weyl-symmetric factor as follows,

$$
\hat{Z}=Z_{\mathrm{Weyl}} \cdot Z, \quad Z_{\mathrm{Weyl}}=\operatorname{PE}\left[\frac{1}{2} \frac{\sinh \frac{m \pm \epsilon_{+}}{2}}{\sinh \frac{\epsilon_{1,2}}{2}} \sum_{\alpha \in \Psi_{\mathfrak{g}}^{+}}\left(e^{\alpha(\mathbf{v})}-e^{-\alpha(\mathbf{v})}\right)\right] .
$$

The reason that we introduce the Weyl symmetric factor is that the elliptic genus expansion $Z=Z_{0} \sum_{\mathfrak{n}} Z_{\mathfrak{n}} e^{-\mathfrak{n} \cdot \mathbf{v}}$ is not symmetric under the Weyl reflection $\mathbf{v} \rightarrow-\mathbf{v}$. By multiplying the prefactor $Z_{\text {Weyl }}$, one can make the full index $\hat{Z}=Z_{\text {Weyl }} Z$ symmetric under the Weyl reflection. Also, the contribution at the $k^{\text {th }}$ fixed point on $S^{5}$ is given by $\hat{Z}_{[k]}=$ $\hat{Z}_{\mathbb{R}^{4} \times T^{2}}\left(\tau_{[k]}, v_{[k]}, \epsilon_{1,[k]}, \epsilon_{2,[k]}, m_{[k]}\right)$, and the chemical potentials are given as follows [74],

$$
\begin{aligned}
& \tau_{[k]}=\frac{2 \pi i}{\omega_{k}}, \quad v_{[k], a}=\frac{2 \pi \omega}{\omega_{k}} v_{a} \\
& \epsilon_{1,[k]}=2 \pi i\left(\frac{\omega_{k+1}}{\omega_{k}}-1\right), \quad \epsilon_{2,[k]}=2 \pi i\left(\frac{\omega_{k-1}}{\omega_{k}}-1\right), \quad m_{[k]}=-\pi i\left(\frac{\Delta_{L}}{\omega_{k}}+1\right) .
\end{aligned}
$$

where $\omega=\frac{\omega_{1}+\omega_{2}+\omega_{3}}{3}$. Second, $S_{0}$ is the classical action of the $5 \mathrm{~d}$ MSYM on the squashed $S^{5}$, and it is given as follows,

$$
S_{0}=\frac{2 \pi^{2} \omega^{2}}{\omega_{1} \omega_{2} \omega_{3}} \sum_{a, b=1}^{r}\left(\Omega^{-1}\right)_{a, b} v_{a} v_{b}
$$

Third, $S_{\text {bkgd }}$ is the background action which gives a divergent behavior when the chemical potentials are small $\Delta_{1,2} \sim \omega_{1,2,3} \ll 1$. Its form is given as follows [77, 82],

$$
S_{\mathrm{bkgd}}=r_{\mathfrak{g}} \frac{\pi^{2}}{24} \frac{\omega_{1}^{2}+\omega_{2}^{2}+\omega_{3}^{2}-2 \omega_{1} \omega_{2}-2 \omega_{2} \omega_{3}-2 \omega_{3} \omega_{1}-\Delta_{L}^{2}}{\omega_{1} \omega_{2} \omega_{3}} .
$$

Also, $E_{0}$ is the Casimir energy which gives a divergent behavior when the chemical potentials are large $\Delta_{1,2} \sim \omega_{1,2,3} \gg 1$. Its form is given as follows [83],

$$
\begin{aligned}
E_{0}= & \frac{h_{G}^{\vee} d_{G}}{384} \frac{\left(\Delta_{R}^{2}-\Delta_{L}^{2}\right)^{2}}{\omega_{1} \omega_{2} \omega_{3}}+r_{G} \frac{\Delta_{R}+\Delta_{L}}{48} \\
& +\frac{r_{G}}{384} \frac{\left(\Delta_{R}-\Delta_{L}\right)\left(2 \omega_{1}-\Delta_{R}-\Delta_{L}\right)\left(2 \omega_{2}-\Delta_{R}-\Delta_{L}\right)\left(2 \omega_{3}-\Delta_{R}-\Delta_{L}\right)}{\omega_{1} \omega_{2} \omega_{3}} .
\end{aligned}
$$

Lastly, $\left|\Omega_{\mathfrak{g}}\right|$ is the determinant of the Cartan matrix, and $W_{\mathfrak{g}}$ is the dimension of the Weyl group of $\mathfrak{g}$. For the simply-laced Lie algebra, they are given as follows,

$$
\begin{array}{llll}
\mathfrak{g}=A_{r}, & \left|\Omega_{\mathfrak{g}}\right|=r+1, & W_{\mathfrak{g}}=(r+1) ! \\
\mathfrak{g}=D_{r}, & \left|\Omega_{\mathfrak{g}}\right|=4, & W_{\mathfrak{g}}=2^{r-1} r ! \\
\mathfrak{g}=E_{6}, & \left|\Omega_{\mathfrak{g}}\right|=3, & W_{\mathfrak{g}}=51,840 \\
\mathfrak{g}=E_{7}, & \left|\Omega_{\mathfrak{g}}\right|=2, & W_{\mathfrak{g}}=2,903,040 \\
\mathfrak{g}=E_{8}, & \left|\Omega_{\mathfrak{g}}\right|=1, & W_{\mathfrak{g}}=696,729,600 .
\end{array}
$$


Although the $6 \mathrm{~d}$ superconformal indices have been studied extensively in various places, the proper prescription to evaluate the integral (4.17) is currently unknown when the chemical potentials are fully refined. The main reason is that the chemical potentials in the integrand of (4.17) is 'S-dualized' in the sense that $\tau_{[k]} \sim \frac{1}{\omega_{k}}$. Therefore, the integral expression (4.17) does not have a proper fugacity expansion structure with respect to $e^{-\omega_{1,2,3}}$ and $e^{-\Delta_{1,2}}$.

Therefore, we will study the index (4.16) with unrefined chemical potentials. Eventually, we would take a single chemical potential to be independent by setting $\Delta_{1}=2 \omega$, $\Delta_{2}=\omega$, and $\omega_{1,2,3}=\omega$. However, directly substituting these unrefined values makes the integrand of (4.17) singular due to the vanishing $\Omega$-background. Therefore, we shall first consider the following unrefinement,

$$
\Delta_{L}=\omega_{1}+\omega_{2}-\omega_{3},
$$

and then take $\omega_{1,2,3} \rightarrow \omega$ limit later. In the above setting, the chemical potentials of each $\mathbb{R}^{4} \times T^{2}$ indices in the integrand satisfy a simple relation. According to (4.19), we obtain

$$
e^{-m_{[1]}}=e^{+\epsilon_{-,[1]}}, \quad e^{-m_{[2]}}=e^{-\epsilon_{-,[2]}}, \quad e^{-m_{[3]}}=e^{+\epsilon_{+,[3]}}
$$

Here, $e^{-m}=e^{ \pm \epsilon_{+}}$and $e^{-m}=e^{ \pm \epsilon_{-}}$are special points that the elliptic genera become much simplified. When $e^{-m}=e^{ \pm \epsilon_{+}}$, all elliptic genera we obtain become 0 unless the base degree is empty. When $e^{-m}=e^{ \pm \epsilon_{-}}$, all elliptic genera we obtain become $+1,-1$, or 0 . For all the data we obtained, we check that the following two properties hold,

$$
\begin{aligned}
e^{-m} & =e^{ \pm \epsilon_{+}}: \sum_{\mathfrak{n}} Z_{\mathfrak{n}} e^{-\mathfrak{n} \cdot \mathbf{v}}=1 \\
e^{-m} & =e^{ \pm \epsilon_{-}}: \sum_{\mathfrak{n}} Z_{\mathfrak{n}} e^{-\mathfrak{n} \cdot \mathbf{v}}=\prod_{\alpha \in \Psi_{\mathfrak{g}}^{+}}\left(1-e^{-\alpha \cdot \mathbf{v}}\right) .
\end{aligned}
$$

According to (4.26), the maximal base degree for the non-vanishing elliptic genus when $e^{-m}=e^{ \pm \epsilon_{-}}$is expected to be $\mathfrak{n}=2 \rho$ where $\rho$ is a Weyl vector (4.10). Unfortunately, we could not reach that bound due to the limited computing power. However, our data in appendix B still provide non-trivial evidence for (4.26) inclulding E-type cases.

Similarly, the pure momentum contribution $Z_{0}$ in (3.8) and the Weyl prefactor in (4.18) become

$$
\begin{aligned}
e^{-m}=e^{ \pm \epsilon_{+}}: & Z_{0}=q^{\frac{r_{\mathfrak{g}}}{24} \eta(\tau)^{-r_{\mathfrak{g}}}} & Z_{\mathrm{Weyl}}=1, \\
e^{-m}=e^{ \pm \epsilon_{-}}: & Z_{0}=1 & Z_{\mathrm{Weyl}}=\prod_{\alpha \in \Psi_{G}^{+}}\left(\frac{1-e^{\alpha(\mathbf{v})}}{1-e^{-\alpha(\mathbf{v})}}\right)^{\frac{1}{2}} .
\end{aligned}
$$

Then, one can rewrite the integral expression (4.17) as follows,

$$
\begin{aligned}
I= & e^{-\frac{h_{\mathfrak{g}}^{\vee} d_{\mathfrak{g}}}{24} \frac{\omega_{3}\left(\omega_{1}+\omega_{2}\right)^{2}}{\omega_{1} \omega_{2}}}\left[e^{-\frac{\omega_{3}}{24}} \eta\left(\frac{2 \pi i}{\omega_{3}}\right)\right]^{-r_{\mathfrak{g}}} \\
& \times \frac{1}{W_{\mathfrak{g}} \sqrt{\left|\Omega_{\mathfrak{g}}\right|}} \int\left[\prod_{a=1}^{r_{\mathfrak{g}}} d v_{a}\right] e^{-S_{0}} \prod_{\alpha \in \Psi_{\mathfrak{g}}^{+}}\left(2 \sinh \frac{\pi \omega \alpha(\mathbf{v})}{\omega_{1}}\right)\left(2 \sinh \frac{\pi \omega \alpha(\mathbf{v})}{\omega_{2}}\right) .
\end{aligned}
$$


Now, the integrand in (4.28) becomes Gaussian, and the evaluation is straightforward. After taking the further unrefinement $\omega_{1,2,3}=\omega$ and using the S-duality of the Dedekind eta function $\eta\left(\frac{2 \pi i}{\omega}\right)=\sqrt{\frac{\omega}{2 \pi}} \eta\left(-\frac{\omega}{2 \pi i}\right)$, we obtain that

$$
I=\operatorname{PE}\left[r_{\mathfrak{g}} \frac{e^{-\omega}}{1-e^{-\omega}}\right] \prod_{\alpha \in \Psi_{\mathfrak{g}}^{+}}\left(1-e^{-\omega \alpha \cdot \rho}\right)
$$

where $\rho=\frac{1}{2} \sum_{\alpha \in \Psi_{\mathfrak{g}}^{+}} \alpha$ is a Weyl vector. The above unrefined index can be also written in the following form,

$$
I=\mathrm{PE}\left[\frac{\sum_{n \in \mathfrak{D}_{\mathfrak{g}}} e^{-n \omega}}{1-e^{-\omega}}\right] .
$$

Here, $\mathfrak{D}_{\mathfrak{g}}$ is a set of dimensions of the Casimir operators of the Lie algebra $\mathfrak{g}$, and it is given as follows,

$$
\begin{array}{rlrl}
\mathfrak{D}_{A_{N}} & =\{2,3, \ldots, N\}, & \mathfrak{D}_{D_{N}} & =\{2,4,6, \ldots, 2 N-2, N\} \\
\mathfrak{D}_{E_{6}} & =\{2,5,6,8,9,12\}, & \mathfrak{D}_{E_{7}}=\{2,6,8,10,12,14,18\}, \\
\mathfrak{D}_{E_{8}} & =\{2,8,12,14,18,20,24,30\} . & &
\end{array}
$$

The unrefined limit corresponds to the 'chiral algebra limit' of the $6 \mathrm{~d}$ theory, and the superconformal index (4.30) is the same with the $\mathcal{W}$-algebra character of type $\mathfrak{g}[73]$. The unrefined superconformal index (4.30) was previously computed for A/D-type $(2,0)$ theories with the instanton partition function method. However, it had been still a conjecture that the index should become the $\mathcal{W}$-algebra character for E-type also. In this paper, we partially check that the conjecture is true by computing the self-dual strings' elliptic genera in $(2,0)$ E-type theories.

\section{Concluding remarks}

In this paper, we studied the elliptic genera of the self-dual strings in general $6 \mathrm{~d}(2,0)$ SCFTs. We found that the elliptic genus is invariant under the flip symmetry, which is a novel kinematical constraint that we found in this paper. Using the $5 \mathrm{~d}$ perturbative data as an input, we obtained elliptic genera at low base degrees using the modular property and the flip symmetry. Then, we could further conjecture the vanishing bound of the GV invariants that enables us to bootstrap the elliptic genera up to arbitrary base degrees.

We did various consistency checks with our results from the bootstrap. For the elliptic genera of $(2,0)$ A-type theories, our result is exactly the same as the localization computation in [19]. The general formulas for the elliptic genera in D/E-type theories are currently unknown. However, we check that our results agree with the instanton partition function of $5 \mathrm{~d}$ maximal SYM with the D-type gauge group [61, 62]. For E-type theories, our results give the first non-perturbative computation beyond the $5 \mathrm{~d}$ perturbative data.

As straightforward applications, we utilize our data to compute the $6 \mathrm{~d}$ Cardy formula on $\mathbb{R}^{4} \times T^{2}[70]$ and the unrefined $(2,0)$ superconformal index, which is previously computed for A, or D-type theories only. There seem to be many other important topics that our results can be used. Here, we finish our paper by listing some of them. 
First, it would be interesting to construct the instanton partition functions of $5 \mathrm{~d}$ E-type maximal SYM from the elliptic genera of the 6d $(2,0)$ E-type SCFT. In general, one can study the instanton partition function from the ADHM construction [84] of the instanton quantum mechanics if the gauge group is classical. Unlike classical groups, computing the instantons of the exceptional gauge group has been a difficult problem. Although instanton partition functions in many $5 \mathrm{~d}$ exceptional gauge theories were computed [30, 41, 45, 85], there has been no known result for the maximal SYM case. However, the string fugacity expansion of the instanton partition function can be computed from our data in appendix B. Therefore, if one can construct an appropriate ansatz for the instanton partition functions of E-type maximal SYM, the coefficients can be fitted by comparing with the elliptic genera.

Second, one can also consider the extension to $6 \mathrm{~d}$ little string theories (LSTs), which are non-local QFTs given by the UV-completion of 6d $(2,0)$ SCFTs, or $(1,1)$ SYMs. They also admit affine ADE-type classification, and the elliptic genera were computed in [86] for A-type LSTs. Especially in [44], the authors bootstrapped the elliptic genera of type LSTs using the T-duality between $(2,0)$ and $(1,1)$ LSTs. However, the T-duality bootstrap is incomplete for D/E-type LSTs. It would be interesting if our flip-symmetry or the vanishing condition for the GV invariants can give non-trivial results for the D/E-type LSTs also.

Third, our result could provide hints to other methods of computing the elliptic genera of $(2,0)$ SCFTs. In particular, it would be nice to see if one could write down $6 \mathrm{~d}$ blow-up equations that the elliptic genera are supposed to satisfy, along the line of [36-39].

Lastly, let us make a few comments on the modular bootstrap of the elliptic genera in $6 \mathrm{~d}(1,0)$ SCFTs. In this paper, we could bootstrap all the elliptic genera in $(2,0)$ SCFTs only from the $5 \mathrm{~d}$ perturbative data and the modular anomaly. However, a similar bootstrap procedure is known to be impossible for general $(1,0)$ SCFTs with gauge groups, unless we know the precise vanishing condition [43]. Still, one can utilize the flip symmetry to bootstrap the elliptic genera in $(1,0)$ theory if it preserves $\mathrm{SU}(2)$ flavor symmetry originating from the $(2,0) \mathrm{SO}(5) \mathrm{R}$-symmetry. If the flip symmetry and other kinematical constraints can fix the elliptic genera of $(1,0)$ SCFTs at low base degrees, it would be helpful to conjecture the precise vanishing bound from them to initiate the bootstrap program.

\section{Acknowledgments}

We are grateful to Kihong Lee, Kimyeong Lee, Amir-Kian Kashani-Poor, Joonho Kim, Seok Kim, and Xin Wang for helpful discussions. We would like to thank Amir-Kian Kashani-Poor, Seok Kim, and Kimyeong Lee for their reading of the draft and providing inspiring comments. In particular, we thank Amir-Kian Kashani-Poor for kindly improving English writing of the draft. JN is supported by KIAS Individual Grant PG076401. ZD is supported by KIAS Individual Grant PG076901. 


\section{A Modular forms}

In this appendix, we collect some standard facts about the theory of modular forms [87-89].

\section{Elliptic modular forms.}

Definition 1. Suppose $k$ is an integer. A function $f: \mathbb{H} \rightarrow \mathbb{C}$ is called a modular form of weight $k$ for full modular group $S L(2, \mathbb{Z})$ if $f$ is holomorphic on $\mathbb{H} \cup\{\infty\}$ and satisfies the following equation

$$
f\left(\frac{a \tau+b}{c \tau+d}\right)=(c \tau+d)^{k} f(\tau), \text { for any }\left(\begin{array}{ll}
a & b \\
c & d
\end{array}\right) \in S L(2, \mathbb{Z})
$$

In particular, if we choose the matrix $T=\left(\begin{array}{ll}1 & 1 \\ 0 & 1\end{array}\right)$, we find that $f(\tau+1)=f(\tau)$. Thus if we introduce $q=\exp (2 \pi i \tau), f$ can be expanded as a power series at $\tau=i \infty$,

$$
f(\tau)=\sum_{n \geq 0} a_{n} q^{n} .
$$

The condition $n \geq 0$ is guaranteed by the holomorphicity at the infinity. If furthermore $a_{n}=0$, then $f$ is called a cusp form.

A natural construction of modular forms involves summing over all two dimensional lattice points, which are reshuffled under the $\operatorname{SL}(2, \mathbb{Z})$ action. Indeed, we can define normalized Eisenstein series,

$$
E_{k}(\tau)=\frac{1}{\zeta(k)} \sum_{\substack{(m, n) \in \mathbb{Z}^{2} \\(m, n) \neq(0,0)}} \frac{1}{(m+n \tau)^{k}}
$$

and it is easy to show that $E_{k}$ is a modular form of weight $k$ when $k$ is an integer larger than two. ${ }^{8}$ When $k$ is odd, it trivially vanishes so we only need to focus on $k$ being even. The overall constant is chosen such that when power expanded in terms of $q$, the leading constant of $E_{k}$ is one. In fact, we have,

$$
E_{k}=1-\frac{2 k}{B_{k}} \sum_{n=1}^{\infty} \sigma_{k-1}(n) q^{n}
$$

where $B_{k}$ is the $k^{\text {th }}$ Bernoulli number and $\sigma_{i}(n)$ denotes the sum of the $i^{\text {th }}$ powers of the positive divisors of $n$.

It is a classical result that the ring of holomorphic (elliptic) modular forms $M_{*}=$ $\oplus_{k \geq 0} M_{k}(\mathrm{SL}(2, \mathbb{Z}))$ is freely generated by $E_{4}$ and $E_{6}[87,88]$.

Functions that are not exactly modular are also important. In the main text, we also need the Dedekind $\eta$ function,

$$
\eta(\tau)=q^{\frac{1}{24}} \prod_{i=1}^{\infty}\left(1-q^{i}\right)
$$

\footnotetext{
${ }^{8}$ This is needed to ensure that we can rearrange the order of summation.
} 
Its $24^{\text {th }}$ power is a cusp modular form of weight twelve. It can be expressed in terms of the two generators,

$$
\eta(\tau)^{24}=\frac{1}{1728}\left(E_{4}(\tau)^{3}-E_{6}(\tau)^{2}\right)=q-24 q^{2}+\cdots
$$

Another important function that is nearly modular is the second Eisenstein series $E_{2}$. Under $\mathrm{SL}(2, \mathbb{Z})$, it transforms as

$$
E_{2}\left(\frac{a \tau+b}{c \tau+d}\right)=(c \tau+d)^{2} E_{2}(\tau)-\frac{6 i}{\pi} c(c \tau+d) .
$$

$E_{2}$ is a famous example of quasimodular forms. In fact, we can enlarge our ring $M_{*}$ to be the ring of quasi-modular forms $\tilde{M}_{*}$, by relaxing the condition (A.1) to have lower powers in $(c \tau+d)$ on the right-hand side. $\tilde{M}_{*}$ is also finitely generated. Actually, it can be shown [87] that $\tilde{M}_{*}$ is generated from $M_{*}$ just by adding one extra generator $E_{2}$.

If one wants to maintain modularity, we can add an extra piece to $E_{2}$ and define

$$
\hat{E}_{2}(\tau)=E_{2}(\tau)-\frac{3}{\pi \operatorname{Im}(\tau)}
$$

$\hat{E}_{2}(\tau)$ can be shown to be modular with weight two, but clearly at the expense of losing holomorphicity. It is an example of almost holomorphic modular forms.

\section{Jacobi modular forms.}

Definition 2. A Jacobi modular form of weight $k$ and index $m$ is a function $\phi: \mathbb{H} \times \mathbb{C} \rightarrow \mathbb{C}$ that depends on a modular parameter $\tau \in \mathbb{H}$ and an elliptic parameter $z \in \mathbb{C}$. It transforms under the action of $S L(2, \mathbb{Z})$ on $\mathbb{H} \times \mathbb{C}$ as

$$
\tau \mapsto \tau_{\gamma}=\frac{a \tau+b}{c \tau+d}, \quad z \mapsto z_{\gamma}=\frac{z}{c \tau+d} \quad \text { with } \quad\left(\begin{array}{ll}
a & b \\
c & d
\end{array}\right) \in \operatorname{SL}(2, \mathbb{Z}),
$$

as

$$
\begin{aligned}
\phi_{k, m}\left(\tau_{\gamma}, z_{\gamma}\right) & =(c \tau+d)^{k} e^{\frac{2 \pi i m c z^{2}}{c \tau+d}} \phi_{k, m}(\tau, z), \\
\phi_{k, m}(\tau, z+\lambda \tau+\mu) & =e^{-2 \pi i m\left(\lambda^{2} \tau+2 \lambda z\right)} \phi_{k, m}(\tau, z)
\end{aligned} \quad \forall \lambda, \mu \in \mathbb{Z},
$$

Eq. (A.10) is known as the modular transformation which is a generalization of modular transform, while the second one (A.11) is known as the elliptic transform.

From the definition, if we choose $T=\left(\begin{array}{ll}1 & 1 \\ 0 & 1\end{array}\right)$ and $\lambda=0, \mu=1$ in equations (A.10) and (A.11) respectively, we see that the Jacobi form is invariant under the shift $\tau \rightarrow \tau+1$ and $z \rightarrow z+1$, hence it enjoys a double Fourier expansion,

$$
\phi(\tau, z)=\sum_{n, r} c(n, r) q^{n} y^{r}, \quad \text { for } \quad q=e^{2 \pi i \tau}, \quad y=e^{2 \pi i z} .
$$

It can be shown that $c(n, r)$ only depends on $r$ and an $\mathrm{SL}(2, \mathbb{Z})$ invariant combination $4 n m-r^{2}$. In other words, $c(n, r)=C\left(4 n m-r^{2}, r\right)$. We can further define three classes 
of Jacobi modular forms: holomorphic Jacobi forms $J_{*, *}^{h}$ satisfy the constraint $c(n, r)=0$ unless $4 n m \geq r^{2}$, cusp forms $J_{*, *}^{c}$ satisfy $c(n, r)=0$ unless $4 n m>r^{2}$ and weak Jacobi forms $J_{*, *}^{w}$ satisfy $c(n, r)=0$ unless $n \geq 0$. Clearly we have,

$$
J_{*, *}^{c} \subset J_{*, *}^{h} \subset J_{*, *}^{w} .
$$

An important theorem in [89] shows that weak Jacobi forms $J_{*, *}^{w}$ of integer index is freely generated over the ring of elliptic modular forms by two generators $\varphi_{-2,1}(\tau, z)$ and $\varphi_{0,1}(\tau, z)$.

$\varphi_{-2,1}$ and $\varphi_{0,1}$ can be defined in terms of the Jacobi theta functions. For $a$ and $b$ $\in\{0,1 / 2\}$, we have

$$
\Theta\left[\begin{array}{l}
a \\
b
\end{array}\right](\tau, z)=\sum_{n \in \mathbb{Z}} e^{\pi i(n+a)^{2} \tau+2 \pi i z(n+a)+2 \pi i b n}
$$

The four theta functions in our convention are

$$
\theta_{1}=i \Theta\left[\begin{array}{c}
\frac{1}{2} \\
\frac{1}{2}
\end{array}\right], \quad \theta_{2}=\Theta\left[\begin{array}{c}
\frac{1}{2} \\
0
\end{array}\right], \quad \theta_{3}=\Theta\left[\begin{array}{l}
0 \\
0
\end{array}\right], \quad \theta_{4}=\Theta\left[\begin{array}{c}
0 \\
\frac{1}{2}
\end{array}\right]
$$

Based on $\theta_{i}(\tau, z)$, we can give explicit forms of our two generators,

$$
\begin{aligned}
\varphi_{-2,1}(\tau, z) & =\frac{\theta_{1}(\tau, z)^{2}}{\eta(\tau)^{6}}, \\
\varphi_{0,1}(\tau, z) & =4\left(\frac{\theta_{2}(\tau, z)^{2}}{\theta_{2}(\tau, 0)^{2}}+\frac{\theta_{3}(\tau, z)^{2}}{\theta_{3}(\tau, 0)^{2}}+\frac{\theta_{4}(\tau, z)^{2}}{\theta_{4}(\tau, 0)^{2}}\right) .
\end{aligned}
$$

We also give the first few terms in their $q$-expansion for convenience,

$$
\begin{aligned}
\phi_{-2,1}(\tau, z) & =-(y-2+1 / y)+2\left(y^{2}-4 y+6-4 / y+1 / y^{2}\right) q+\cdots, \\
\phi_{0,1}(\tau, z) & =(y+10+1 / y)+\left(10 y^{2}-64 y+108-64 / y+10 / y^{2}\right) q+\ldots
\end{aligned}
$$

Finally, we point out an interesting relation between Jacobi forms $\phi(\tau, z)$ and quasimodular forms $E_{2}$. Looking back to the modular transformation (A.10), the exponential factor on the right-hand side looks a bit annoying, but actually one can introduce an automorphic correction to cancel it. Recall the modular transformation of $E_{2}$ (A.7), it is easy to verify that the combination $\Phi(\tau, z)=e^{\frac{m \pi^{2}}{3} E_{2} z^{2}} \phi(\tau, z)$ transforms without that factor. In other words, if we consider a series expansion of $\Phi(\tau, z)$ in $z$,

$$
\Phi(\tau, z)=\sum_{n \geq 0} g_{n}(\tau) z^{n}
$$

Each $g_{n}(\tau)$ now behaves nicely as genuine elliptic modular forms. From this we can extract a differential equation for $\phi(\tau, z)$,

$$
\frac{\partial}{\partial E_{2}} \Phi(\tau, z)=0 \Rightarrow\left(\frac{\partial}{\partial E_{2}}+\frac{m \pi^{2}}{3} z^{2}\right) \phi(\tau, z)=0 .
$$




\section{B Elliptic genera}

In this section, we summarize the elliptic genera of the ADE M-strings of a few base degrees. We consider base degrees with $n_{i} \neq 0$ for all $1 \leq i \leq r_{\mathfrak{g}}$ so that the full chain does not degenerate into the shorter pieces of the M-string chain. We used a shorthand notation such that

$$
\varphi_{-n, 1}\left(\frac{\epsilon_{+}}{2 \pi i}\right)=R_{n}, \quad \varphi_{-n, 1}\left(\frac{\epsilon_{-}}{2 \pi i}\right)=L_{n}, \quad \varphi_{-n, 1}\left(\frac{m}{2 \pi i}\right)=f_{n} .
$$

Also, we further use the identity that

$$
\frac{1}{12}\left(\varphi_{-2,1}\left(\frac{x}{2 \pi i}\right) \varphi_{0,1}\left(\frac{y}{2 \pi i}\right)-\varphi_{-2,1}\left(\frac{y}{2 \pi i}\right) \varphi_{0,1}\left(\frac{x}{2 \pi i}\right)\right)=\theta(x \pm y)
$$

where we defined a function $\theta(x)$ as follows,

$$
\theta(x) \equiv \frac{\theta_{1}\left(\tau, \frac{x}{2 \pi i}\right)}{\eta(\tau)^{3}}
$$

and use a notation that $\theta(x \pm y)=\theta(x+y) \theta(x-y)$. Finally, in the case when the numerator of a higher rank elliptic genus is factorized into that of a lower rank elliptic genus, we write it as (higher rank base degree) $=$ factor $\times$ (lower rank base degree) in the tables. We find that attaching a single M-string to another single M-string yields $\theta\left(m \pm \epsilon_{-}\right)$factor universally. 


\begin{tabular}{|c|c|}
\hline $\mathfrak{n}$ & $\mathcal{N}$ \\
\hline$(1$ & $\theta\left(m \pm \epsilon_{+}\right)$ \\
\hline$(2)$ & $\frac{1}{2^{11} 3^{6}} \theta\left(m \pm \epsilon_{+}\right) \theta\left(m \pm \epsilon_{-}\right)\left(27 R_{2}^{6} E_{4}^{3}-45 R_{2}^{4} R_{0}^{2} E_{4}^{2}-24 R_{2}^{5} R_{0} E_{4} E_{6}-15 R_{2}^{2} R_{0}^{4} E_{4}-32 R_{2}^{6} E_{6}^{2}-40 R_{2}^{3} R_{0}^{3} E_{6}+R_{0}^{6}\right)$ \\
\hline$(3)^{*}$ & 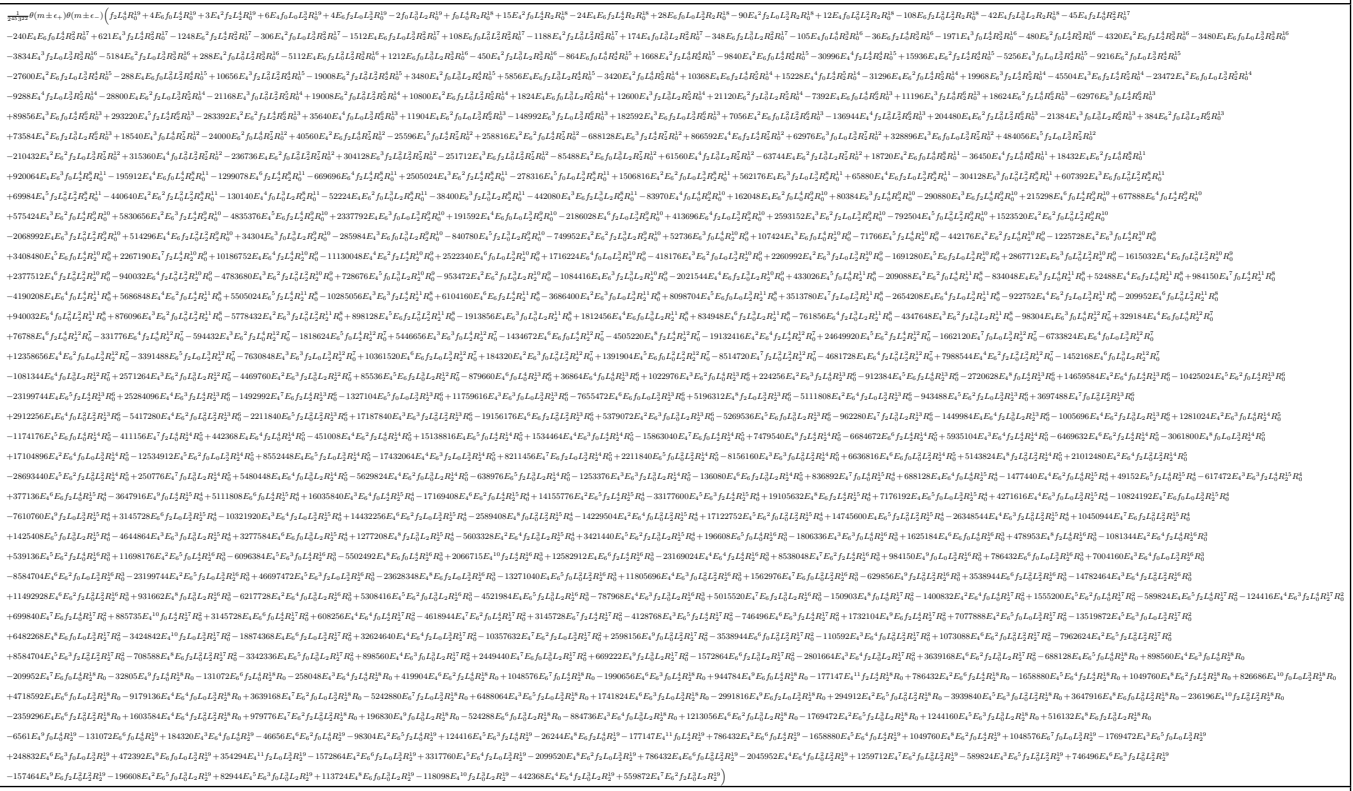 \\
\hline$(1)$ & $\theta\left(m \pm \epsilon_{+}\right) \cdot \theta\left(m \pm \epsilon_{-}\right)$ \\
\hline 1 & $\begin{array}{l}\frac{1}{2^{13} 3^{7}} \theta\left(m \pm \epsilon_{+}\right)^{2}\left(-54 f_{2} L_{2}^{3} R_{2}^{3} R_{0} E_{4}{ }^{3}-27 f_{0} L_{2}^{3} R_{2}^{4} E_{4}{ }^{3}+81 f_{2} L_{0} L_{2}^{2} R_{2}^{4} E_{4}{ }^{3}+18 f_{2} L_{2}^{3} R_{2} R_{0}^{3} E_{4}{ }^{2}+36 f_{0} L_{2}^{3} R_{2}^{2} R_{0}^{2} E_{4}{ }^{2}\right. \\
+18 f_{0} L_{0} L_{2}^{2} R_{2}^{3} R_{0} E_{4}{ }^{2}-54 f_{2} L_{0}^{2} L_{2} R_{2}^{3} R_{0} E_{4}{ }^{2}-9 f_{2} L_{0}^{3} R_{2}^{4} E_{4}{ }^{2}-9 f_{0} L_{0}^{2} L_{2} R_{2}^{4} E_{4}{ }^{2}+24 f_{2} L_{2}^{3} R_{2}^{2} R_{0}^{2} E_{4} E_{6}+24 f_{0} L_{2}^{3} R_{2}^{3} R_{0} E_{4} E_{6} \\
-24 f_{2} L_{0} L_{2}^{2} R_{2}^{3} R_{0} E_{4} E_{6}-24 f_{2} L_{0}^{2} L_{2} R_{2}^{4} E_{4} E_{6}+3 f_{0} L_{2}^{3} R_{0}^{4} E_{4}+3 f_{2} L_{0} L_{2}^{2} R_{0}^{4} E_{4}+18 f_{0} L_{0} L_{2}^{2} R_{2} R_{0}^{3} E_{4}-6 f_{2} L_{0}^{2} L_{2} R_{2} R_{0}^{3} E_{4}-12 f_{2} L_{0}^{3} R_{2}^{2} R_{0}^{2} E_{4} \\
-6 f_{0} L_{0}^{3} R_{2}^{3} R_{0} E_{4}+64 f_{2} L_{2}^{3} R_{2}^{3} R_{0} E_{6}{ }^{2}+32 f_{0} L_{2}^{3} R_{2}^{4} E_{6}{ }^{2}-96 f_{2} L_{0} L_{2}^{2} R_{2}^{4} E_{6}{ }^{2}+4 f_{2} L_{2}^{3} R_{0}^{4} E_{6}+20 f_{0} L_{2}^{3} R_{2} R_{0}^{3} E_{6}+12 f_{2} L_{0} L_{2}^{2} R_{2} R_{0}^{3} E_{6}+36 f_{0} L_{0} L_{2}^{2} R_{2}^{2} R_{0}^{2} E_{6} \\
\left.-36 f_{2} L_{0}^{2} L_{2} R_{2}^{2} R_{0}^{2} E_{6}-20 f_{2} L_{0}^{3} R_{2}^{3} R_{0} E_{6}-12 f_{0} L_{0}^{2} L_{2} R_{2}^{3} R_{0} E_{6}-4 f_{0} L_{0}^{3} R_{2}^{4} E_{6}+f_{2} L_{0}^{3} R_{0}^{4}-3 f_{0} L_{0}^{2} L_{2} R_{0}^{4}+2 f_{0} L_{0}^{3} R_{2} R_{0}^{3}\right)\end{array}$ \\
\hline$\left(\begin{array}{lll}1 & 1 & 1\end{array}\right)$ & $\theta\left(m \pm \epsilon_{+}\right) \cdot \theta\left(m \pm \epsilon_{-}\right)^{2}$ \\
\hline$\left(\begin{array}{lll}2 & 1 & 1\end{array}\right)$ & $\theta\left(m \pm \epsilon_{-}\right) \times\left(\begin{array}{ll}2 & 1\end{array}\right)$ \\
\hline$\left(\begin{array}{lll}1 & 2 & 1\end{array}\right)$ & $\begin{array}{l}\frac{1}{2^{13} 3^{7}} \theta\left(m \pm \epsilon_{+}\right)^{2} \theta\left(m \pm \epsilon_{-}\right)\left(108 f_{2} L_{2}^{3} L_{0} R_{2}^{3} E_{4}{ }^{3}-27 f_{0} L_{2}^{4} R_{2}^{3} E_{4}{ }^{3}-81 f_{2} L_{2}^{4} R_{0} R_{2}^{2} E_{4}{ }^{3}-36 f_{2} L_{2} L_{0}^{3} R_{2}^{3} E_{4}{ }^{2}-18 f_{0} L_{2}^{2} L_{0}^{2} R_{2}^{3} E_{4}{ }^{2}\right. \\
-54 f_{2} L_{2}^{2} L_{0}^{2} R_{0} R_{2}^{2} E_{4}{ }^{2}+36 f_{0} L_{2}^{3} L_{0} R_{0} R_{2}^{2} E_{4}{ }^{2}+36 f_{2} L_{2}^{3} L_{0} R_{0}^{2} R_{2} E_{4}{ }^{2}+9 f_{2} L_{2}^{4} R_{0}^{3} E_{4}{ }^{2}+27 f_{0} L_{2}^{4} R_{0}^{2} R_{2} E_{4}{ }^{2}-48 f_{2} L_{2}^{2} L_{0}^{2} R_{2}^{3} E_{4} E_{6} \\
+24 f_{0} L_{2}^{4} R_{0} R_{2}^{2} E_{4} E_{6}+24 f_{2} L_{2}^{4} R_{0}^{2} R_{2} E_{4} E_{6}-3 f_{0} L_{0}^{4} R_{2}^{3} E_{4}-9 f_{2} L_{0}^{4} R_{0} R_{2}^{2} E_{4}-12 f_{0} L_{2} L_{0}^{3} R_{0} R_{2}^{2} E_{4}-12 f_{2} L_{2} L_{0}^{3} R_{0}^{2} R_{2} E_{4}+6 f_{2} L_{2}^{2} L_{0}^{2} R_{0}^{3} E_{4} \\
+18 f_{0} L_{2}^{2} L_{0}^{2} R_{0}^{2} R_{2} E_{4}+12 f_{0} L_{2}^{3} L_{0} R_{0}^{3} E_{4}-128 f_{2} L_{2}^{3} L_{0} R_{2}^{3} E_{6}{ }^{2}+32 f_{0} L_{2}^{4} R_{2}^{3} E_{6}{ }^{2}+96 f_{2} L_{2}^{4} R_{0} R_{2}^{2} E_{6}{ }^{2}-8 f_{2} L_{0}^{4} R_{2}^{3} E_{6}-16 f_{0} L_{2} L_{0}^{3} R_{2}^{3} E_{6}-48 f_{2} L_{2} L_{0}^{3} R_{0} R_{2}^{2} E_{6} \\
\left.+16 f_{2} L_{2}^{3} L_{0} R_{0}^{3} E_{6}+48 f_{0} L_{2}^{3} L_{0} R_{0}^{2} R_{2} E_{6}+8 f_{0} L_{2}^{4} R_{0}^{3} E_{6}+f_{2} L_{0}^{4} R_{0}^{3}+3 f_{0} L_{0}^{4} R_{0}^{2} R_{2}-4 f_{0} L_{2} L_{0}^{3} R_{0}^{3}\right)\end{array}$ \\
\hline
\end{tabular}

Table 3. Elliptic genera of $A_{1,2,3}$. The result that cannot be obtained from the flip symmetry is marked with the asterisk. 


\begin{tabular}{|c|c|}
\hline $\mathfrak{n}$ & $\mathcal{N}$ \\
\hline$\left(\begin{array}{ccc} & 1 & \\
1 & 1 & 1\end{array}\right)$ & $\theta\left(m \pm \epsilon_{+}\right) \cdot \theta\left(m \pm \epsilon_{-}\right)^{3}$ \\
\hline$\left(\begin{array}{lll} & 1 & \\
2 & 1 & 1\end{array}\right)$ & $\theta\left(m \pm \epsilon_{-}\right)^{2} \times\left(\begin{array}{ll}2 & 1\end{array}\right)$ \\
\hline$\left(\begin{array}{lll}1 & 2 & 1\end{array}\right)$ & 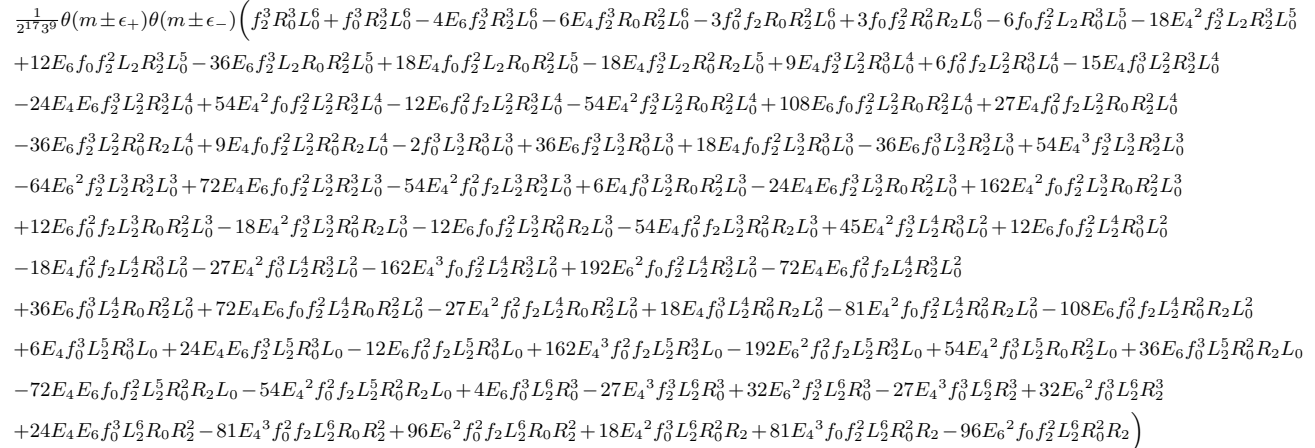 \\
\hline$\left(\begin{array}{lll} & 1 & \\
2 & 2 & 1\end{array}\right)$ & 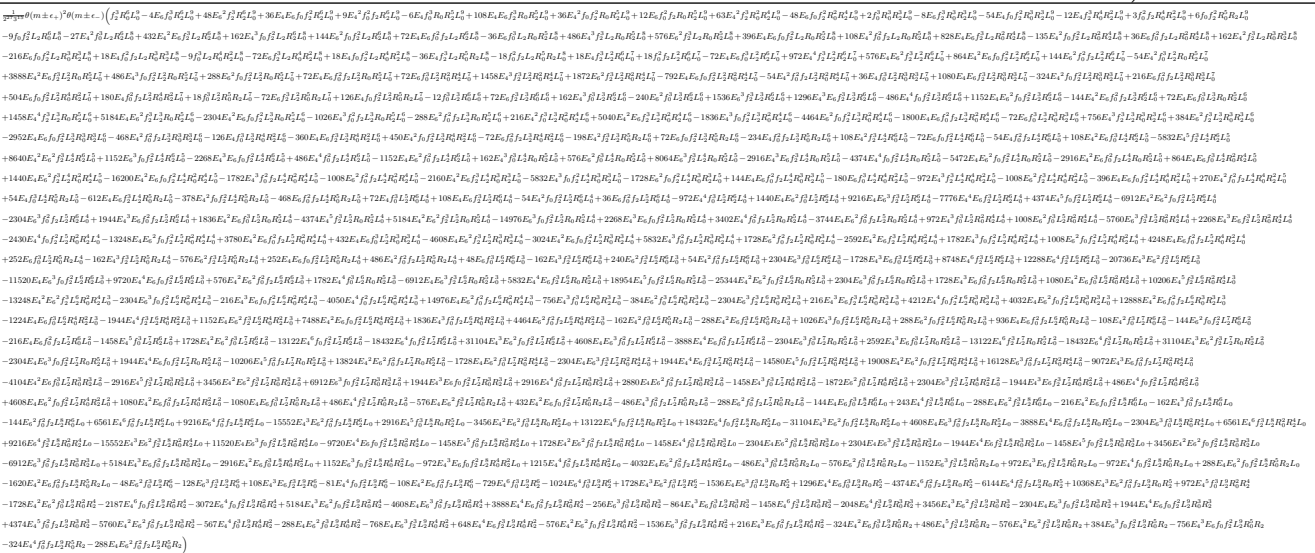 \\
\hline
\end{tabular}

Table 4. Elliptic genera of $D_{4}$. The result that cannot be obtained from the flip symmetry is marked with the asterisk. 


\begin{tabular}{|c|c|}
\hline $\mathfrak{n}$ & $\mathcal{N}$ \\
\hline 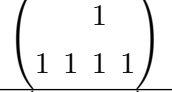 & $\theta\left(m \pm \epsilon_{+}\right) \cdot \theta\left(m \pm \epsilon_{-}\right)^{4}$ \\
\hline$\left(\begin{array}{lllll} & & & 1 \\
1 & 1 & 1 & 2 \\
& & & 1 \\
& & & 1 & \\
2 & 1 & 1\end{array}\right)$ & $\theta\left(m \pm \epsilon_{-}\right)^{3} \times\left(\begin{array}{ll}2 & 1\end{array}\right)$ \\
\hline$\left(\begin{array}{llll} & & 1 \\
1 & 2 & 1 & 1\end{array}\right)$ & $\theta\left(m \pm \epsilon_{-}\right)^{2} \times\left(\begin{array}{lll}1 & 2 & 1\end{array}\right)$ \\
\hline 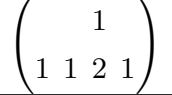 & 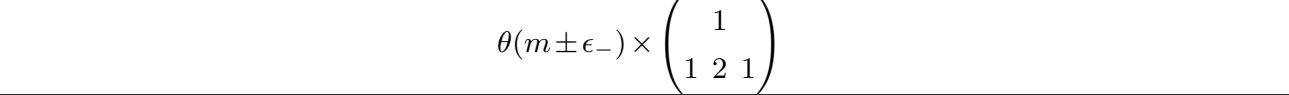 \\
\hline 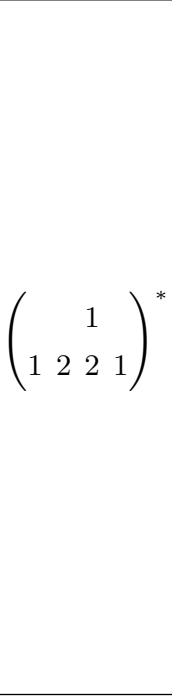 & 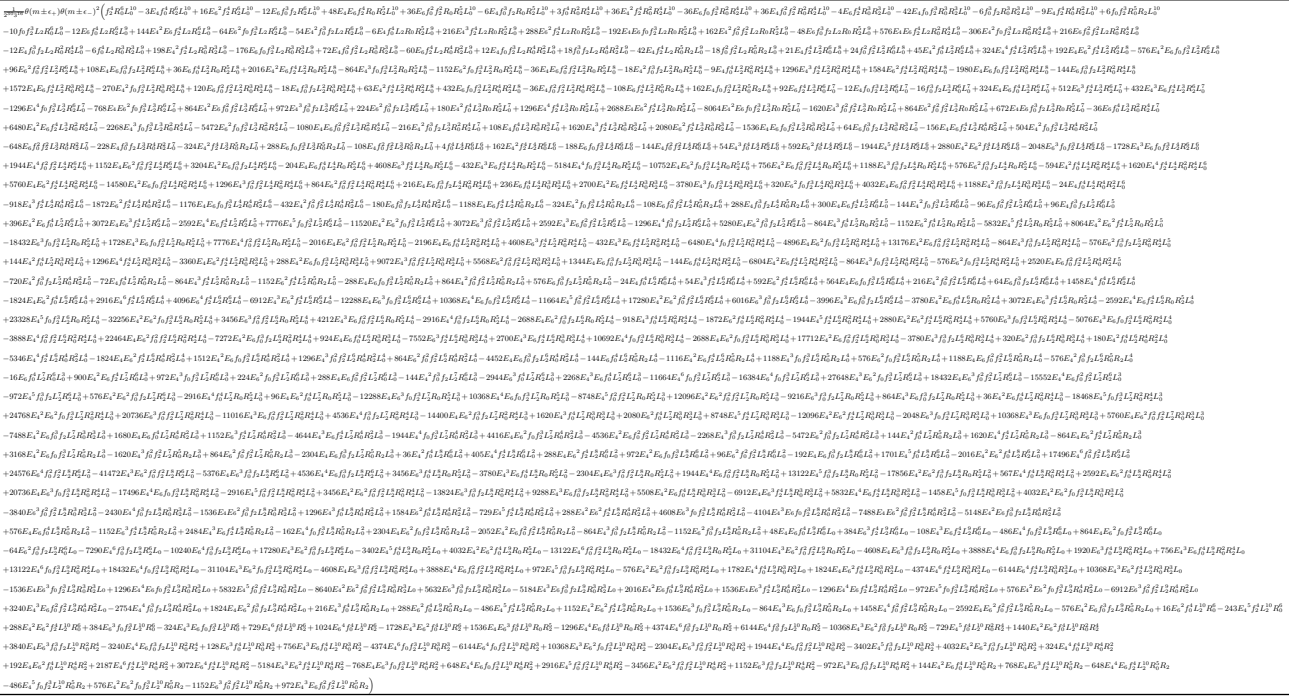 \\
\hline
\end{tabular}

Table 5. Elliptic genera of $D_{5}$. The result that cannot be obtained from the flip symmetry is marked with the asterisk. 


\begin{tabular}{|c|c|}
\hline $\mathfrak{n}$ & $\mathcal{N}$ \\
\hline$\left(\begin{array}{ccccc} & \multicolumn{3}{c}{1} & \\
\\
1 & 1 & 1 & 1 & 1\end{array}\right)$ & $\theta\left(m \pm \epsilon_{+}\right) \cdot \theta\left(m \pm \epsilon_{-}\right)^{5}$ \\
\hline 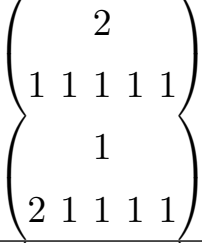 & $\theta\left(m \pm \epsilon_{-}\right)^{4} \times\left(\begin{array}{ll}2 & 1\end{array}\right)$ \\
\hline 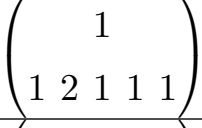 & $\theta\left(m \pm \epsilon_{-}\right)^{3} \times\left(\begin{array}{lll}1 & 2 & 1\end{array}\right)$ \\
\hline$\left(\begin{array}{ccccc} & & 1 & & \\
1 & 1 & 2 & 1 & 1\end{array}\right)$ & $\theta\left(m \pm \epsilon_{-}\right)^{2} \times\left(\begin{array}{ccc}1 \\
1 & 2 & 1\end{array}\right)$ \\
\hline
\end{tabular}

Table 6. Elliptic genera of $E_{6}$.

\begin{tabular}{|c|c|}
\hline $\mathfrak{n}$ & $\mathcal{N}$ \\
\hline$\left(\begin{array}{cccccc} & & 1 & & & \\
1 & 1 & 1 & 1 & 1 & 1\end{array}\right)$ & $\theta\left(m \pm \epsilon_{+}\right) \cdot \theta\left(m \pm \epsilon_{-}\right)^{6}$ \\
\hline 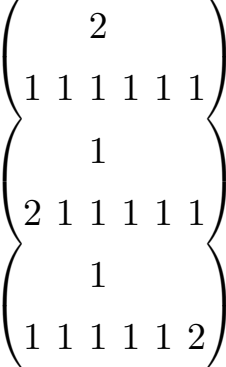 & $\theta\left(m \pm \epsilon_{-}\right)^{5} \times\left(\begin{array}{ll}2 & 1\end{array}\right)$ \\
\hline$\left(\begin{array}{lllllll} & & & 1 & & & \\
1 & 2 & 1 & 1 & 1 & 1 \\
& & & 1 & & & \\
& & 1 & 1 & 2 & 1 & 1\end{array}\right)$ & $\theta\left(m \pm \epsilon_{-}\right)^{4} \times\left(\begin{array}{lll}1 & 2 & 1\end{array}\right)$ \\
\hline$\left(\begin{array}{cccccc} & & 1 & & & \\
1 & 1 & 2 & 1 & 1 & 1\end{array}\right)$ & $\theta\left(m \pm \epsilon_{-}\right)^{3} \times\left(\begin{array}{ccc}1 \\
1 & 2 & 1\end{array}\right)$ \\
\hline
\end{tabular}

Table 7. Elliptic genera of $E_{7}$. 


\begin{tabular}{|c|c|}
\hline $\mathfrak{n}$ & $\mathcal{N}$ \\
\hline$\left(\begin{array}{ccccccc} & & & & & & \\
1 & 1 & 1 & 1 & 1 & 1 & 1\end{array}\right)$ & $\theta\left(m \pm \epsilon_{+}\right) \cdot \theta\left(m \pm \epsilon_{-}\right)^{7}$ \\
\hline 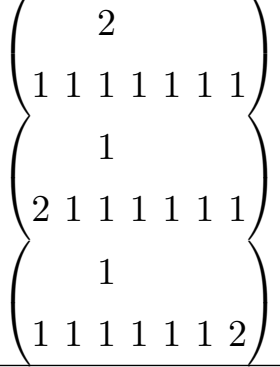 & $\theta\left(m \pm \epsilon_{-}\right)^{6} \times\left(\begin{array}{ll}2 & 1\end{array}\right)$ \\
\hline$\left(\begin{array}{llllllll} & & 1 & & & & \\
1 & 2 & 1 & 1 & 1 & 1 & 1 \\
& & 1 & & & & \\
1 & 1 & 1 & 2 & 1 & 1 & 1\end{array}\right)$ & $\theta\left(m \pm \epsilon_{-}\right)^{5} \times\left(\begin{array}{lll}1 & 2 & 1\end{array}\right)$ \\
\hline$\left(\begin{array}{ccccccc} & 1 & & & & \\
1 & 1 & 2 & 1 & 1 & 1 & 1\end{array}\right)$ & 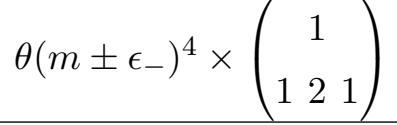 \\
\hline
\end{tabular}

Table 8. Elliptic genera of $E_{8}$. 


\begin{tabular}{|c|cccc|}
\hline$\{(1,1,2,1,1,1), e, 2\}$ & $e=1$ & 2 & 3 & 4 \\
\hline$g=0$ & -158 & -14198 & -630198 & -18294632 \\
1 & 34 & 8323 & 605826 & 24543838 \\
2 & & -1506 & -224344 & -13830246 \\
3 & & 84 & 40334 & 4281922 \\
4 & & & -3640 & -801172 \\
5 & & & 140 & 92994 \\
\hline
\end{tabular}

\begin{tabular}{|c|cccc|}
\hline$\{(1,1,2,1,1,1), e, 3\}$ & $e=1$ & 2 & 3 & 4 \\
\hline$g=0$ & 51 & 6090 & 311415 & 9876000 \\
1 & -8 & -3098 & -274390 & -12459206 \\
2 & & 477 & 91798 & 6540187 \\
3 & & -24 & -14764 & -1868740 \\
4 & & & 1190 & 319875 \\
5 & & & -40 & -33634 \\
\hline
\end{tabular}

\begin{tabular}{|c|cccc|}
\hline$\{(1,1,2,1,1,1), e, 7\}$ & $e=1$ & 2 & 3 & 4 \\
\hline$g=0$ & & -38 & -5266 & -296958 \\
1 & & 5 & 2498 & 252881 \\
2 & & & -370 & -82390 \\
3 & & & 18 & 13096 \\
4 & & & & -1046 \\
5 & & & & 34 \\
\hline
\end{tabular}

Table 9. Unrefined GV invariants from $(2,0) E_{6}$ SCFT at base degree $(1,1,2,1,1,1)$, fiber degree 1 to 4 and mass degree 2,3 and 7 .

\section{GV invariants}

Here, we list some GV invariants of geometries that engineer E-type $(2,0)$ SCFTs, extracted from the elliptic genera. 


\begin{tabular}{|c|ccccc|}
\hline$\{(1,1,2,1,1,1), 1,1\}$ & $2 j_{+}=0$ & 1 & 2 & 3 & 4 \\
\hline $2 j_{-}=0$ & 55 & & 28 & & 1 \\
1 & & 33 & & 4 & \\
\hline
\end{tabular}

\begin{tabular}{|c|cccccc|}
\hline$\{(1,1,2,1,1,1), 2,2\}$ & $2 j_{+}=0$ & 1 & 2 & 3 & 4 & 5 \\
\hline $2 j_{-}=0$ & & 1305 & & 315 & & 6 \\
1 & 900 & & 740 & & 71 & \\
2 & & 301 & & 94 & & 4 \\
3 & 6 & & 16 & & 6 & \\
\hline
\end{tabular}

Table 10. Refined GV invariants from $(2,0) E_{6}$ SCFT at base degree $(1,1,2,1,1,1)$, fiber degree 1, 2 with mass degree 1 and 2 respectively.

\begin{tabular}{|c|cccc|}
\hline$\{(1,1,2,1,1,1,1), e, 1\}$ & $e=1$ & 2 & 3 & 4 \\
\hline$g=0$ & 323 & 27832 & 1319194 & 42422226 \\
1 & -86 & -17200 & -1289146 & -56879466 \\
2 & & 3244 & 482499 & 31860566 \\
3 & & -168 & -86166 & -9715442 \\
4 & & & 7490 & 1768586 \\
5 & & & -280 & -197964 \\
\hline
\end{tabular}

\begin{tabular}{|c|cccc|}
\hline$\{(1,1,2,1,1,1,1), e, 4\}$ & $e=1$ & 2 & 3 & 4 \\
\hline$g=0$ & -10 & -2216 & -166600 & -7098368 \\
1 & 1 & 868 & 124584 & 7922736 \\
2 & & -94 & -33822 & -3580000 \\
3 & & 3 & 4175 & 851158 \\
4 & & & -240 & -116466 \\
5 & & & 5 & 9321 \\
\hline
\end{tabular}

\begin{tabular}{|c|cccc|}
\hline$\{(1,1,2,1,1,1,1), e, 5\}$ & $e=1$ & 2 & 3 & 4 \\
\hline$g=0$ & 1 & 438 & 45561 & 2346531 \\
1 & & -118 & -28154 & -2317034 \\
2 & & 7 & 5970 & 902530 \\
3 & & & -528 & -178930 \\
4 & & & 17 & 19447 \\
5 & & & & -1122 \\
\hline
\end{tabular}

Table 11. Unrefined GV invariants from $(2,0) E_{7}$ SCFT at base degree $(1,1,2,1,1,1,1)$, fiber degree 1 to 4 and mass degree 1,4 and 5 . 


\begin{tabular}{|c|ccccc|}
\hline$\{(1,1,2,1,1,1,1), 1,1\}$ & $2 j_{+}=0$ & 1 & 2 & 3 & 4 \\
\hline $2 j_{-}=0$ & 59 & & 29 & & 1 \\
1 & & 35 & & 4 & \\
\hline
\end{tabular}

\begin{tabular}{|c|cccc|}
\hline$\{(1,1,2,1,1,1,1), 2,4\}$ & $2 j_{+}=0$ & 1 & 2 & 3 \\
\hline $2 j_{-}=0$ & & 346 & & 54 \\
1 & 201 & & 111 & \\
2 & & 30 & & 4 \\
3 & 0 & & 1 & \\
\hline
\end{tabular}

Table 12. Refined GV invariants from $(2,0) E_{7}$ SCFT at base degree $(1,1,2,1,1,1,1)$, fiber degree 1 and 2 with mass degree 1 and 4 respectively.

\begin{tabular}{|c|c|c|c|c|}
\hline$\{(1,1,1,1,1,1,1,1), e, 3\}$ & $e=2$ & 3 & 4 & 5 \\
\hline$g=0$ & 1100 & 57254 & 1851595 & 44039385 \\
\hline 1 & -378 & -37522 & -1771932 & -55325096 \\
\hline 2 & 21 & 7462 & 611859 & 27249080 \\
\hline 3 & & -406 & -88228 & -6517634 \\
\hline 4 & & & 4256 & 745339 \\
\hline 5 & & & & -31626 \\
\hline$\{(1,1,1,1,1,1,1,1), e, 4\}$ & $e=2$ & 3 & 4 & 5 \\
\hline$g=0$ & -224 & -18256 & -731712 & -19082144 \\
\hline 1 & 42 & 9604 & 617120 & 22839016 \\
\hline 2 & & -1344 & -179592 & -10100384 \\
\hline 3 & & 35 & 20146 & 2097858 \\
\hline 4 & & & -630 & -197036 \\
\hline 5 & & & & 6146 \\
\hline$\{(1,1,1,1,1,1,1,1), e, 5\}$ & $e=2$ & 3 & 4 & 5 \\
\hline$g=0$ & 21 & 3948 & 216538 & 6995758 \\
\hline 1 & & -1470 & -153062 & -7192724 \\
\hline 2 & & 105 & 34650 & 2743685 \\
\hline 3 & & & -2590 & -466144 \\
\hline 4 & & & 35 & 32480 \\
\hline 5 & & & & -602 \\
\hline
\end{tabular}

Table 13. Unrefined GV invariants from $(2,0) E_{8}$ SCFT at base degree $(1,1,1,1,1,1,1,1)$, fiber degree 2 to 5 and mass degree 3,4 and 5 . 


\begin{tabular}{|c|c|c|c|c|}
\hline \multicolumn{2}{|c|}{$(1,1,1,1,1,1,1,1), 0,0$} & \multicolumn{3}{|c|}{$2 j_{+}=0 \quad 1$} \\
\hline \multicolumn{2}{|c|}{$2 j_{-}=0$} & \multicolumn{3}{|c|}{1} \\
\hline \multicolumn{2}{|c|}{$(1,1,1,1,1,1,1,1), 1,1$} & $j_{+}=$ & & 2 \\
\hline \multicolumn{2}{|l|}{$2 j_{-}=0$} & 29 & & 8 \\
\hline \multicolumn{2}{|l|}{1} & \multicolumn{3}{|c|}{15} \\
\hline$\{(1,1,1,1,1,1,1,1), 2,2\}$ & & \multicolumn{2}{|c|}{$2 j_{+}=0 \quad 1$} \\
\hline $2 j_{-}=0$ & \multicolumn{4}{|c|}{443} \\
\hline 1 & \multirow{2}{*}{\multicolumn{2}{|c|}{351}} & & 169 \\
\hline 2 & & & & \\
\hline
\end{tabular}

Table 14. Refined GV invariants from $(2,0) E_{8}$ SCFT at base degree $(1,1,1,1,1,1,1,1)$, fiber degree 0,1 and 2 with mass degree 0,1 and 2 respectively.

Open Access. This article is distributed under the terms of the Creative Commons Attribution License (CC-BY 4.0), which permits any use, distribution and reproduction in any medium, provided the original author(s) and source are credited.

\section{References}

[1] D. Gaiotto, $N=2$ dualities, JHEP 08 (2012) 034 [arXiv:0904.2715] [INSPIRE].

[2] L.F. Alday, D. Gaiotto and Y. Tachikawa, Liouville correlation functions from four-dimensional gauge theories, Lett. Math. Phys. 91 (2010) 167 [arXiv:0906.3219] [INSPIRE].

[3] T. Dimofte, D. Gaiotto and S. Gukov, Gauge theories labelled by three-manifolds, Commun. Math. Phys. 325 (2014) 367 [arXiv:1108.4389] [InSPIRE].

[4] A. Gadde, S. Gukov and P. Putrov, Fivebranes and 4-manifolds, Prog. Math. 319 (2016) 155 [arXiv: 1306.4320] [INSPIRE].

[5] E. Witten, Some comments on string dynamics, in Strings '95: future perspectives in string theory, (1995), pg. 501 [hep-th/9507121] [INSPIRE].

[6] N. Seiberg and E. Witten, Comments on string dynamics in six-dimensions, Nucl. Phys. B 471 (1996) 121 [hep-th/9603003] [INSPIRE].

[7] C. Vafa, Evidence for F-theory, Nucl. Phys. B 469 (1996) 403 [hep-th/9602022] [InSPIRE].

[8] D.R. Morrison and C. Vafa, Compactifications of F-theory on Calabi-Yau threefolds. 1, Nucl. Phys. B 473 (1996) 74 [hep-th/9602114] [INSPIRE].

[9] D.R. Morrison and C. Vafa, Compactifications of F-theory on Calabi-Yau threefolds. 2, Nucl. Phys. B 476 (1996) 437 [hep-th/9603161] [INSPIRE].

[10] J.J. Heckman, D.R. Morrison and C. Vafa, On the classification of $6 D$ SCFTs and generalized ADE orbifolds, JHEP 05 (2014) 028 [Erratum ibid. 06 (2015) 017] [arXiv:1312.5746] [INSPIRE]. 
[11] J.J. Heckman, D.R. Morrison, T. Rudelius and C. Vafa, Atomic classification of $6 D$ SCFTs, Fortsch. Phys. 63 (2015) 468 [arXiv:1502.05405] [inSPIRE].

[12] S.H. Katz, A. Klemm and C. Vafa, Geometric engineering of quantum field theories, Nucl. Phys. B 497 (1997) 173 [hep-th/9609239] [INSPIRE].

[13] N.A. Nekrasov, Seiberg-Witten prepotential from instanton counting, Adv. Theor. Math. Phys. 7 (2003) 831 [hep-th/0206161] [INSPIRE].

[14] N. Nekrasov and A. Okounkov, Seiberg-Witten theory and random partitions, Prog. Math. 244 (2006) 525 [hep-th/0306238] [INSPIRE].

[15] T.J. Hollowood, A. Iqbal and C. Vafa, Matrix models, geometric engineering and elliptic genera, JHEP 03 (2008) 069 [hep-th/0310272] [INSPIRE].

[16] A. Iqbal, C. Kozcaz and C. Vafa, The refined topological vertex, JHEP 10 (2009) 069 [hep-th/0701156] [INSPIRE].

[17] M. Aganagic, M.C.N. Cheng, R. Dijkgraaf, D. Krefl and C. Vafa, Quantum geometry of refined topological strings, JHEP 11 (2012) 019 [arXiv:1105.0630] [INSPIRE].

[18] M.-X. Huang, A.-K. Kashani-Poor and A. Klemm, The $\Omega$ deformed B-model for rigid $N=2$ theories, Annales Henri Poincaré 14 (2013) 425 [arXiv:1109.5728] [INSPIRE].

[19] B. Haghighat, A. Iqbal, C. Kozçaz, G. Lockhart and C. Vafa, M-strings, Commun. Math. Phys. 334 (2015) 779 [arXiv: 1305.6322] [INSPIRE].

[20] B. Haghighat, G. Lockhart and C. Vafa, Fusing E-strings to heterotic strings: $E+E \rightarrow H$, Phys. Rev. D 90 (2014) 126012 [arXiv:1406.0850] [INSPIRE].

[21] W. Cai, M.-X. Huang and K. Sun, On the elliptic genus of three E-strings and heterotic strings, JHEP 01 (2015) 079 [arXiv:1411.2801] [INSPIRE].

[22] F. Benini, R. Eager, K. Hori and Y. Tachikawa, Elliptic genera of two-dimensional $N=2$ gauge theories with rank-one gauge groups, Lett. Math. Phys. 104 (2014) 465 [arXiv: 1305.0533] [INSPIRE].

[23] F. Benini, R. Eager, K. Hori and Y. Tachikawa, Elliptic genera of $2 d N=2$ gauge theories, Commun. Math. Phys. 333 (2015) 1241 [arXiv:1308.4896] [INSPIRE].

[24] B. Haghighat, C. Kozcaz, G. Lockhart and C. Vafa, Orbifolds of M-strings, Phys. Rev. D 89 (2014) 046003 [arXiv: 1310.1185] [INSPIRE].

[25] B. Haghighat, A. Klemm, G. Lockhart and C. Vafa, Strings of minimal 6d SCFTs, Fortsch. Phys. 63 (2015) 294 [arXiv:1412.3152] [INSPIRE].

[26] J. Kim, S. Kim, K. Lee, J. Park and C. Vafa, Elliptic genus of E-strings, JHEP 09 (2017) 098 [arXiv: 1411.2324] [INSPIRE].

[27] A. Gadde, B. Haghighat, J. Kim, S. Kim, G. Lockhart and C. Vafa, 6d string chains, JHEP 02 (2018) 143 [arXiv: 1504.04614] [INSPIRE].

[28] J. Kim, S. Kim and K. Lee, Higgsing towards E-strings, JHEP 01 (2021) 110 [arXiv: 1510.03128] [INSPIRE].

[29] H.-C. Kim, S. Kim and J. Park, 6d strings from new chiral gauge theories, arXiv: 1608.03919 [INSPIRE].

[30] H.-C. Kim, J. Kim, S. Kim, K.-H. Lee and J. Park, 6d strings and exceptional instantons, Phys. Rev. D 103 (2021) 025012 [arXiv: 1801.03579] [InSPIRE]. 
[31] S.-S. Kim, M. Taki and F. Yagi, Tao probing the end of the world, PTEP 2015 (2015) 083B02 [arXiv: 1504.03672] [INSPIRE].

[32] H. Hayashi and K. Ohmori, 5d/6d DE instantons from trivalent gluing of web diagrams, JHEP 06 (2017) 078 [arXiv: 1702.07263] [INSPIRE].

[33] O. Foda and R.-D. Zhu, An elliptic topological vertex, J. Phys. A 51 (2018) 465401 [arXiv: 1805.12073] [INSPIRE].

[34] M.-X. Huang, A. Klemm and M. Poretschkin, Refined stable pair invariants for E-, $M$ - and [p,q]-strings, JHEP 11 (2013) 112 [arXiv:1308.0619] [INSPIRE].

[35] B. Haghighat, A. Klemm, G. Lockhart and C. Vafa, Strings of minimal 6d SCFTs, Fortsch. Phys. 63 (2015) 294 [arXiv:1412.3152] [INSPIRE].

[36] J. Gu, B. Haghighat, K. Sun and X. Wang, Blowup equations for 6d SCFTs. Part I, JHEP 03 (2019) 002 [arXiv: 1811.02577] [INSPIRE].

[37] J. Gu, A. Klemm, K. Sun and X. Wang, Elliptic blowup equations for 6d SCFTs. Part II. Exceptional cases, JHEP 12 (2019) 039 [arXiv: 1905.00864] [INSPIRE].

[38] J. Gu, B. Haghighat, A. Klemm, K. Sun and X. Wang, Elliptic blowup equations for $6 d$ SCFTs. Part III. E-strings, M-strings and chains, JHEP 07 (2020) 135 [arXiv:1911.11724] [INSPIRE].

[39] J. Gu, B. Haghighat, A. Klemm, K. Sun and X. Wang, Elliptic blowup equations for $6 d$ SCFTs. Part IV. Matters, arXiv:2006.03030 [INSPIRE].

[40] D.R. Morrison and W. Taylor, Classifying bases for $6 D$ F-theory models, Central Eur. J. Phys. 10 (2012) 1072 [arXiv:1201.1943] [INSPIRE].

[41] M. Del Zotto and G. Lockhart, On exceptional instanton strings, JHEP 09 (2017) 081 [arXiv:1609.00310] [INSPIRE].

[42] J. Gu, M.-X. Huang, A.-K. Kashani-Poor and A. Klemm, Refined BPS invariants of $6 d$ SCFTs from anomalies and modularity, JHEP 05 (2017) 130 [arXiv:1701.00764] [INSPIRE].

[43] M. Del Zotto, J. Gu, M.-X. Huang, A.-K. Kashani-Poor, A. Klemm and G. Lockhart, Topological strings on singular elliptic Calabi-Yau 3-folds and minimal 6d SCFTs, JHEP 03 (2018) 156 [arXiv : 1712.07017] [INSPIRE].

[44] J. Kim, K. Lee and J. Park, On elliptic genera of 6d string theories, JHEP 10 (2018) 100 [arXiv: 1801.01631] [INSPIRE].

[45] M. Del Zotto and G. Lockhart, Universal features of BPS strings in six-dimensional SCFTs, JHEP 08 (2018) 173 [arXiv:1804.09694] [INSPIRE].

[46] Z. Duan, J. Gu and A.-K. Kashani-Poor, Computing the elliptic genus of higher rank E-strings from genus $0 G W$ invariants, JHEP 03 (2019) 078 [arXiv:1810.01280] [INSPIRE].

[47] B. Haghighat, W. Yan and S.-T. Yau, ADE string chains and mirror symmetry, JHEP 01 (2018) 043 [arXiv: 1705.05199] [inSPIRE].

[48] H. Shimizu and Y. Tachikawa, Anomaly of strings of $6 d N=(1,0)$ theories, JHEP 11 (2016) 165 [arXiv: 1608.05894] [inSPIRE].

[49] N. Bobev, M. Bullimore and H.-C. Kim, Supersymmetric Casimir energy and the anomaly polynomial, JHEP 09 (2015) 142 [arXiv: 1507.08553] [INSPIRE].

[50] R. Gopakumar and C. Vafa, $M$ theory and topological strings. 1, hep-th/9809187 [INSPIRE]. 
[51] R. Gopakumar and C. Vafa, $M$ theory and topological strings. 2, hep-th/9812127 [INSPIRE].

[52] R. Gopakumar and C. Vafa, On the gauge theory/geometry correspondence, AMS/IP Stud. Adv. Math. 23 (2001) 45 [Adv. Theor. Math. Phys. 3 (1999) 1415] [hep-th/9811131] [INSPIRE].

[53] A. Klemm, P. Mayr and C. Vafa, BPS states of exceptional noncritical strings, Nucl. Phys. B Proc. Suppl. 58 (1997) 177 [hep-th/9607139] [INSPIRE].

[54] M. Bershadsky, S. Cecotti, H. Ooguri and C. Vafa, Holomorphic anomalies in topological field theories, Nucl. Phys. B 405 (1993) 279 [hep-th/9302103] [INSPIRE].

[55] M. Bershadsky, S. Cecotti, H. Ooguri and C. Vafa, Kodaira-Spencer theory of gravity and exact results for quantum string amplitudes, Commun. Math. Phys. 165 (1994) 311 [hep-th/9309140] [INSPIRE].

[56] M. Aganagic, V. Bouchard and A. Klemm, Topological strings and (almost) modular forms, Commun. Math. Phys. 277 (2008) 771 [hep-th/0607100] [INSPIRE].

[57] M.-X. Huang, S. Katz and A. Klemm, Topological string on elliptic CY 3-folds and the ring of Jacobi forms, JHEP 10 (2015) 125 [arXiv:1501.04891] [INSPIRE].

[58] B. Haghighat, S. Murthy, C. Vafa and S. Vandoren, F-theory, spinning black holes and multi-string branches, JHEP 01 (2016) 009 [arXiv: 1509.00455] [INSPIRE].

[59] H.-C. Kim, S. Kim, E. Koh, K. Lee and S. Lee, On instantons as Kaluza-Klein modes of M5-branes, JHEP 12 (2011) 031 [arXiv:1110.2175] [INSPIRE].

[60] M. Bullimore, H.-C. Kim and P. Koroteev, Defects and quantum Seiberg-Witten geometry, JHEP 05 (2015) 095 [arXiv: 1412.6081] [INSPIRE].

[61] C. Hwang, J. Kim, S. Kim and J. Park, General instanton counting and 5d SCFT, JHEP 07 (2015) 063 [Addendum ibid. 04 (2016) 094] [arXiv:1406.6793] [INSPIRE].

[62] Y. Hwang, J. Kim and S. Kim, M5-branes, orientifolds, and S-duality, JHEP 12 (2016) 148 [arXiv: 1607.08557] [INSPIRE].

[63] S. Choi, J. Kim, S. Kim and J. Nahmgoong, Large AdS black holes from QFT, arXiv: 1810.12067 [INSPIRE].

[64] J. Kim, S. Kim and J. Song, A 4d N=1 Cardy formula, JHEP 01 (2021) 025 [arXiv: 1904.03455] [INSPIRE].

[65] J. Nahmgoong, 6d superconformal Cardy formulas, arXiv:1907.12582 [INSPIRE].

[66] S.H. Katz, A. Klemm and C. Vafa, $M$ theory, topological strings and spinning black holes, Adv. Theor. Math. Phys. 3 (1999) 1445 [hep-th/9910181] [InSPIRE].

[67] R. Pandharipande and R.P. Thomas, Curve counting via stable pairs in the derived category, Invent. Math. 178 (2009) 407 [arXiv:0707.2348] [INSPIRE].

[68] S.H. Katz, Gromov-Witten invariants via algebraic geometry, Nucl. Phys. B Proc. Suppl. 46 (1996) 108 [hep-th/9510218] [INSPIRE].

[69] J. Choi, S. Katz and A. Klemm, The refined BPS index from stable pair invariants, Commun. Math. Phys. 328 (2014) 903 [arXiv:1210.4403] [INSPIRE].

[70] K. Lee and J. Nahmgoong, Cardy limits of 6d superconformal theories, arXiv:2006.10294 [INSPIRE]. 
[71] S. Choi, J. Kim, S. Kim and J. Nahmgoong, Comments on deconfinement in AdS/CFT, arXiv: 1811.08646 [INSPIRE].

[72] S. Kim and J. Nahmgoong, Asymptotic M5-brane entropy from S-duality, JHEP 12 (2017) 120 [arXiv: 1702.04058] [INSPIRE].

[73] C. Beem, L. Rastelli and B.C. van Rees, $\mathcal{W}$ symmetry in six dimensions, JHEP 05 (2015) 017 [arXiv: 1404.1079] [INSPIRE].

[74] S. Kim and K. Lee, Indices for 6 dimensional superconformal field theories, J. Phys. A 50 (2017) 443017 [arXiv: 1608.02969] [INSPIRE].

[75] H.-C. Kim and S. Kim, M5-branes from gauge theories on the 5-sphere, JHEP 05 (2013) 144 [arXiv:1206.6339] [INSPIRE].

[76] J. Kallen, J.A. Minahan, A. Nedelin and M. Zabzine, $N^{3}$-behavior from $5 D$ Yang-Mills theory, JHEP 10 (2012) 184 [arXiv:1207.3763] [INSPIRE].

[77] H.-C. Kim, J. Kim and S. Kim, Instantons on the 5-sphere and M5-branes, arXiv: 1211.0144 [INSPIRE].

[78] G. Lockhart and C. Vafa, Superconformal partition functions and non-perturbative topological strings, JHEP 10 (2018) 051 [arXiv:1210.5909] [INSPIRE].

[79] H.-C. Kim and K. Lee, Supersymmetric M5 brane theories on $R \times C P^{2}$, JHEP 07 (2013) 072 [arXiv: 1210.0853] [INSPIRE].

[80] H.-C. Kim, S. Kim, S.-S. Kim and K. Lee, The general M5-brane superconformal index, arXiv: 1307.7660 [INSPIRE].

[81] J.A. Minahan, A. Nedelin and M. Zabzine, 5D super Yang-Mills theory and the correspondence to $A d S_{7} / C F T_{6}$, J. Phys. A 46 (2013) 355401 [arXiv:1304.1016] [InSPIRE].

[82] C.-M. Chang, M. Fluder, Y.-H. Lin and Y. Wang, Proving the $6 d$ Cardy formula and matching global gravitational anomalies, arXiv:1910.10151 [INSPIRE].

[83] N. Bobev, M. Bullimore and H.-C. Kim, Supersymmetric Casimir energy and the anomaly polynomial, JHEP 09 (2015) 142 [arXiv: 1507.08553] [INSPIRE].

[84] M.F. Atiyah, N.J. Hitchin, V.G. Drinfeld and Y.I. Manin, Construction of instantons, Phys. Lett. A 65 (1978) 185 [inSPIRE].

[85] J. Kim, S.-S. Kim, K.-H. Lee, K. Lee and J. Song, Instantons from blow-up, JHEP 11 (2019) 092 [Erratum ibid. 06 (2020) 124] [arXiv: 1908.11276] [INSPIRE].

[86] J. Kim, S. Kim and K. Lee, Little strings and T-duality, JHEP 02 (2016) 170 [arXiv: 1503.07277] [INSPIRE].

[87] J.H. Bruinier, G. van der Geer, G. Harder and D. Zagier, The 1-2-3 of modular forms, Springer, Berlin, Heidelberg, Germany (2008).

[88] J.-P. Serre, A course in arithmetic, Springer, New York, NY, U.S.A. (1973).

[89] M. Eichler and D. Zagier, The theory of Jacobi forms, Birkhäuser, Boston, MA, U.S.A. (1985). 